\title{
Concentration phenomena for solutions of superlinear elliptic problems ${ }^{\text {is }}$
}

\section{Phénomènes de concentration pour les solutions de problèmes elliptiques surlinéaires}

\author{
Riccardo Molle ${ }^{\mathrm{a}, *}$, Donato Passaseo ${ }^{\mathrm{b}}$ \\ a Dipartimento di Matematica, Università di Roma "Tor Vergata”, Via della Ricerca Scientifica n. 1, 00133 Roma, Italy \\ b Dipartimento di Matematica “E. De Giorgi”, Università di Lecce, P.O. Box 193, 73100 Lecce, Italy
}

Received 29 July 2004; received in revised form 13 January 2005; accepted 2 February 2005

Available online 20 April 2005

\begin{abstract}
In this paper we look for positive solutions of the problem $-\Delta u+\lambda u=u^{p-1}$ in $\Omega, u=0$ on $\partial \Omega$, where $\Omega$ is a bounded domain in $\mathbb{R}^{n}, n \geqslant 3, p>2$ and $\lambda$ is a positive parameter. We describe new concentration phenomena, which occur as $\lambda \rightarrow+\infty$, and exploit them to construct (for $\lambda$ large enough) positive solutions that concentrate near spheres of codimension 2 as $\lambda \rightarrow+\infty$; these spheres approach the boundary of $\Omega$ as $\lambda \rightarrow+\infty$. Notice that the existence and multiplicity results we obtain hold also in contractible domains arbitrarily close to starshaped domains (no solution can exist if $p \geqslant \frac{2 n}{n-2}$ and $\Omega$ is starshaped, because of Pohožaev's identity). The method we use is completely variational and based on a blow up analysis in the equivariant setting. In order to avoid concentration phenomena near points and to overcome some difficulties related to the lack of compactness, we first modify the nonlinear term in a suitable region, then we solve the modified problem by minimizing the related energy functional on a suitable infinite dimensional manifold and, finally, we show that the solutions of the modified problem solve also our problem, for $\lambda$ large enough, because they are localized in the prescribed region where the nonlinear term has not been modified.
\end{abstract}

(C) 2006 L'Association Publications de l'Institut Henri Poincaré. Published by Elsevier B.V. All rights reserved

\section{Résumé}

Nous démontrons l'existence de solutions positives pour le problème $-\Delta u+\lambda u=u^{p-1}$ en $\Omega, u=0$ sur $\partial \Omega$, où $\Omega$ est un domaine borné de $\mathbb{R}^{n}, n \geqslant 3, p>2$ et $\lambda>0$. Nous décrivons de nouveaux phénomènes de concentration qui apparaissent quand $\lambda \rightarrow+\infty$. Grâce à ceux-ci nous construisons des solutions positives pour $\lambda$ assez grand donc qui se concentrent près

\footnotetext{
Work supported by the Italian national research project "Metodi variazionali e topologici nello studio di fenomeni non lineari".

* Corresponding author.

E-mail address: molle@mat.uniroma2.it (R. Molle).
} 
des sphères de codimention 2 quand $\lambda \rightarrow+\infty$; ces sphères approchent du bord de omega quand $\lambda \rightarrow+\infty$. Il faut remarquer que l'existence de solutions est prouvée pour des domaines qui peuvent être arbitrairement proches de domaines étoilés (quand $p \geqslant \frac{2 n}{n-2}$ et $\Omega$ est étoilé il n'y a pas de solutions, ce qui se déduit de l'identité de Pohožaev). La méthode que nous suivons pour la démonstration est complètement variationnelle. Pour surmonter les difficultés liées à la présence d'opérateurs non compacts, d'abord nous modifions le terme non linéaire; ensuite nous trouvons des solutions du problème modifié en minimisant la fonctionnelle de l'énergie sur une varieté de dimension infinie; enfin nous démontrons que les solutions du problème modifié sont aussi solutions du problème original, parce-qu'elles sont localisées, dans la région où le terme non linéaire n'a pas été modifié.

() 2006 L'Association Publications de l'Institut Henri Poincaré. Published by Elsevier B.V. All rights reserved

MSC: $35 \mathrm{~J} 20 ; 35 \mathrm{~J} 60 ; 35 \mathrm{~J} 65$

Keywords: Variational methods; Lack of compactness; Concentration phenomena; Blow up analysis; Nearly starshaped domains

\section{Introduction}

Let us consider the following problem

$$
\begin{cases}-\Delta u+\lambda u=u^{p-1} & \text { in } \Omega, \\ u>0 & \text { in } \Omega, \\ u=0 & \text { on } \partial \Omega,\end{cases}
$$

where $\Omega$ is a bounded domain of $\mathbb{R}^{n}, n \geqslant 3, p>2$ and $\lambda$ is a positive parameter.

In [13] and [15] this problem has been studied in the case $p=\frac{2 n}{n-2}$ (the critical Sobolev exponent). In this case some concentration phenomena occur when $\lambda \rightarrow 0$ : the solutions tend to concentrate near points of the domain. This fact is used in $[13,15]$ to obtain, for $\lambda>0$ small enough, an arbitrarily large number of positive solutions, having an arbitrarily large number of spikes, under suitable assumptions on the domain $\Omega$.

In the present paper we point out other concentration phenomena, which occur, for all $p>2$, as $\lambda \rightarrow+\infty$ and exploit them to construct positive solutions, for $\lambda>0$ large enough, in the same domains considered in $[13,15]$. Notice that, while the solutions obtained in $[13,15]$ concentrate and blow-up as $\lambda \rightarrow 0$ near a finite number of points, the solutions we construct in the present paper concentrate near spheres of codimension 2 . In $[13,15]$ we proved that for all positive integer $k$ there exist, for $\lambda>0$ small enough, solutions blowing-up as $\lambda \rightarrow 0$ at exactly $k$ points, which approach the boundary of $\Omega$ as $k \rightarrow \infty$; the solutions we obtain in this paper, for $\lambda$ large enough, concentrate near spheres which approach the boundary of $\Omega$ as $\lambda \rightarrow+\infty$ and the rate of concentration is greater than the rate of approaching the boundary. In [13,15] the basic tool is a finite dimensional reduction method of Lyapunov-Schmidt type; here we use a completely variational method. Thus, it is clear that the concentration phenomena and the methods we used in $[13,15]$ are deeply different in nature with respect to the ones we use in the present paper; however, they allow us to state existence and multiplicity results in the same domains (see Examples 2.2 and Remark 2.3) which may be also contractible and even arbitrarily close to starshaped domains in the sense we described in [13] (it is well known that the problem cannot have solutions if $\Omega$ is starshaped and $p \geqslant \frac{2 n}{n-2}$, as a consequence of the Pohožaev's identity: see $[22,4,5])$.

Notice that, on the contrary, Dancer and Zhang (see [7]) obtained nonexistence results for supercritical problems in some domains which are nearly starshaped in a different sense with respect to [13] (see also [19,20,25] for other nonexistence results concerning supercritical problems in nonstarshaped domains).

It is worth pointing out that also for $\lambda=0$ some concentration phenomena occur, related to the exponent $p$. When $p \rightarrow \frac{2 n}{n-2}$, we have concentration near points and this fact has been used to describe the effect of the domain's shape on the existence and the multiplicity of solutions (see [1,2,6,23]) and to construct multispike solutions when $p$ is sufficiently close to $\frac{2 n}{n-2}$ (see, for example, $[12,14]$ and the references therein). When $p$ is not close to $\frac{2 n}{n-2}$, concentration may occur not only near points but also near some more complex sets; for example, in [17] we prove that, for $\lambda=0$ and $p$ large enough, there exist solutions which concentrate near spheres as $p \rightarrow+\infty$. 
The domains we consider in this paper have radial symmetry with respect to the $x_{n}$-axis (see condition (2.1)) and we look for solutions having the same symmetry. The method we use is completely variational and the problem reduces to minimizing the corresponding functional constrained on a suitable manifold. However, let us remark that we do not apply this method directly to problem (1.1): we first modify in a suitable region the nonlinear term, then we solve the modified problem and, finally, we show that the solutions of the modified problem solve also problem (1.1) for $\lambda$ large enough, because they are localized in the prescribed region where the nonlinear term has not been modified. This modification of the nonlinear term is due to several reasons, specially when $\Omega$ meets the $x_{n}$-axis. In fact (see Remark 3.12) if we try to apply the minimizing arguments without having modified the nonlinear term, we see that the minimum is not achieved if $p \geqslant \frac{2 n}{n-2}$ (because of concentration phenomena near points of the $x_{n}$-axis) while, for $p<\frac{2 n}{n-2}$, the minimum is achieved but the minimum points give solutions which concentrate, as $\lambda \rightarrow+\infty$, near points of the $x_{n}$-axis, not near spheres (solutions of this type would not be interesting because already well known).

Finally, let us remark that (unlike the finite dimensional reduction methods of Lyapunov-Schmidt type) our construction does not need to know the limit profile of the solution $u_{\lambda}$; however, the method we use suggests that, suitably rescaled, the function $w_{\lambda}(\rho, \tau)=u_{\lambda}(\rho, 0, \ldots, 0, \tau)$ converges as $\lambda \rightarrow+\infty$ to the unique solution of problem

$$
\begin{cases}-\Delta v+v=v^{p-1}, & v>0 \text { in } \mathbb{R}^{2} \\ v \in H^{1,2}\left(\mathbb{R}^{2}\right), & v(0)=\max _{\mathbb{R}^{2}} v\end{cases}
$$

(see Remark 3.11 for more details).

\section{Statement of the main results and examples}

Let us consider a bounded domain $\Omega$ of $\mathbb{R}^{n}$, satisfying the following symmetry condition

$$
x=\left(x_{1}, \ldots, x_{n}\right) \in \Omega \quad \Longleftrightarrow \quad\left(\rho(x), 0, \ldots, 0, x_{n}\right) \in \Omega,
$$

where $\rho(x)=\left(\sum_{i=1}^{n-1} x_{i}^{2}\right)^{1 / 2}$. We say that a function $u$ defined in $\Omega$ has radial symmetry with respect to the $x_{n}$-axis if $u(x)=u\left(\rho(x), 0, \ldots, 0, x_{n}\right)$ for all $x \in \Omega$.

Let us set

$$
\Sigma(\Omega)=\left\{(\rho, \tau) \in \mathbb{R}^{2}: \rho>0,(\rho, 0, \ldots, 0, \tau) \in \Omega\right\}
$$

and denote by $H_{S}(\Omega)$ the subspace of $H_{0}^{1,2}(\Omega)$ consisting of the functions having radial symmetry with respect to the $x_{n}$-axis.

Theorem 2.1. Let $\Omega$ be a bounded domain of $\mathbb{R}^{n}, n \geqslant 3$, satisfying condition (2.1) and assume that there exists an open subset $A$ of $\mathbb{R}^{2}$ such that

$$
0<\inf \left\{\rho(x): x \in \Omega,\left(\rho(x), x_{n}\right) \in A\right\}<\inf \left\{\rho(x): x \in \Omega,\left(\rho(x), x_{n}\right) \in \partial A\right\} .
$$

Also assume $p>2$. Then, there exists $\bar{\lambda}>0$ such that, for all $\lambda \geqslant \bar{\lambda}$, problem (1.1) has a solution $u_{\lambda} \in H_{S}(\Omega)$.

For all $\lambda \geqslant \bar{\lambda}, u_{\lambda}$ is a bounded smooth function and there exists $c_{\lambda}=\left(\rho_{\lambda}, \tau_{\lambda}\right) \in \Sigma(\Omega)$ such that, for all $\eta>0$, we have

$$
\lim _{\lambda \rightarrow+\infty} \frac{1}{\lambda^{p-2}} \sup \left\{u_{\lambda}(x): x \in \Omega,\left(\rho(x), x_{n}\right) \notin B\left(c_{\lambda}, \eta\right)\right\}=0,
$$

while

$$
\sup _{\Omega} u_{\lambda} \geqslant \lambda^{p-2} \quad \forall \lambda \geqslant \bar{\lambda}
$$


Furthermore, $c_{\lambda} \in A$ for $\lambda>0$ large enough and

$$
\lim _{\lambda \rightarrow+\infty} \rho_{\lambda}=\inf \left\{\rho(x): x \in \Omega,\left(\rho(x), x_{n}\right) \in A\right\} .
$$

Moreover,

$$
\begin{aligned}
& \limsup _{\lambda \rightarrow+\infty} \lambda^{-1 /(p-2)}\left\|u_{\lambda}\right\|_{H_{0}^{1,2}(\Omega)}<+\infty, \\
& \limsup _{\lambda \rightarrow+\infty} \lambda^{(p-4) /(p-2)}\left\|u_{\lambda}\right\|_{L^{2}(\Omega)}^{2}<+\infty, \\
& \limsup _{\lambda \rightarrow+\infty} \lambda^{-2 /(p-2)}\left\|u_{\lambda}\right\|_{L^{p}(\Omega)}^{p}<+\infty .
\end{aligned}
$$

The proof is reported in Section 3.

Examples 2.2. It is clear that, because of the behaviour of the solution $u_{\lambda}$ as $\lambda \rightarrow+\infty$, for $\lambda$ large enough Theorem 2.1 guarantees the existence of $k$ distinct solutions if the domain $\Omega$ satisfies condition (2.3) with respect to $k$ open subsets $A_{1}, \ldots, A_{k}$ pairwise disjoint. For example, in domains $\Omega$ such that

$$
\bar{\Omega}=\left\{\left(x_{1}, \ldots, x_{n}\right) \in \mathbb{R}^{n}: a \leqslant x_{n} \leqslant b, \rho_{1}\left(x_{n}\right) \leqslant \rho(x) \leqslant \rho_{2}\left(x_{n}\right)\right\}
$$

for suitable $a, b$ in $\mathbb{R}, a<b$, and $\rho_{1}, \rho_{2}$ nonnegative functions in $[a, b]$, the number of distinct solutions, for $\lambda$ large enough, is related to the number of local (strict) minimum points of the function $\rho_{1}$, with positive minimum values. This fact allows us to construct examples of domains with trivial topology (even contractible) where the number of solutions is arbitrarily large. For example, let us consider, for all $k \in \mathbb{N}, r>1$ and $s>0$, the domain

$$
\Omega_{r, k}^{s}=\left\{x \in \mathbb{R}^{n}: 1<|x|<r, \rho(x)>s x_{n}, \operatorname{dist}\left(x, C_{m}^{s}\right)>1 \text { for } m=1, \ldots, k-1\right\},
$$

where

$$
C_{m}^{s}=\left\{x \in \mathbb{R}^{n}: \rho(x)=3 m, x_{n}=\frac{3 m}{s}\right\} .
$$

If $r>\frac{3 k}{s}\left(1+s^{2}\right)^{1 / 2}$, Theorem 2.1 applies with $k$ pairwise disjoint open subsets $A_{1}, \ldots, A_{k}$ and guarantees, for $\lambda$ large enough, the existence of $k$ distinct solutions which concentrate, as $\lambda \rightarrow+\infty$, near $k$ distinct $(n-2)$ dimensional spheres of the boundary of $\Omega_{r, k}^{s}$. Notice that similar domains have been also considered in [12-14, $16,17,21]$, where concentration phenomena of different type have been used to obtain existence and multiplicity results.

Remark 2.3. The example of the domain $\Omega_{r, k}^{s}$ (see (2.11)) is particularly significant when $p \geqslant \frac{2 n}{n-2}$. In fact, in this case problem (1.1) has no solution for $\lambda \geqslant 0$ if $\Omega$ is a starshaped domain, as a consequence of the well known Pohožaev's identity. The domains of the form $\Omega_{r, k}^{s}$ allow us to show that problem (1.1), for $\lambda>0$ large enough, can have an arbitrarily large number of solutions in domains arbitrarily close to starshaped domains in a sense (introduced in [13]) we describe below (notice that a different definition of nearly starshaped domain has been given in [7] in order to extend Pohožaev nonexistence result).

For each smooth bounded domain $\Omega$ in $\mathbb{R}^{n}$, let us set

$$
\sigma(\Omega)=\sup _{x_{0} \in \Omega} \inf \left\{v(x) \cdot \frac{x-x_{0}}{\left|x-x_{0}\right|}: x \in \partial \Omega\right\}
$$

where $v(x)$ denotes the outward normal to $\partial \Omega$ in $x$. We say that $\Omega$ is nearly starshaped if $\sigma(\Omega)^{-}=$ $\max \{0,-\sigma(\Omega)\}$ is small $\left(\delta\right.$-nearly-starshaped if $\left.\sigma(\Omega)^{-} \leqslant \delta\right)$. 
Since $\Omega_{r, k}^{s}$ is not a smooth domain, let us consider its neighbourhood

$$
\mathcal{N}_{\eta}\left(\Omega_{r, k}^{s}\right)=\left\{x \in \mathbb{R}^{n}: \operatorname{dist}\left(x, \Omega_{r, k}^{s}\right)<\eta\right\} .
$$

Then, if $\eta \in] 0,1\left[, \mathcal{N}_{\eta}\left(\Omega_{r, k}^{s}\right)\right.$ is a smooth domain for $r$ and $s$ large enough, $\lim _{r, s \rightarrow+\infty} \sigma\left(\mathcal{N}_{\eta}\left(\Omega_{r, k}^{s}\right)\right)=0$ and Theorem 2.1 guarantees the existence of $k$ distinct solutions for $\lambda$ large enough.

\section{Preliminary results and proof of the main theorem}

Notice that, for all $\lambda>0$, a function $u \in H_{0}^{1,2}(\Omega)$ solves the equation $-\Delta u+\lambda u=u^{p-1}$ if and only if the function $\lambda^{-1 /(p-2)} u$ solves the equation $-\lambda^{-1} \Delta u+u=u^{p-1}$. Therefore, if we set $\varepsilon=\lambda^{-1 / 2}$, finding solutions of problem (1.1) for large $\lambda>0$ is equivalent to finding solutions, for small $\varepsilon$, of the following problem

$$
\begin{cases}-\varepsilon^{2} \Delta u+u=u^{p-1} & \text { in } \Omega \\ u>0 & \text { in } \Omega, \\ u=0 & \text { on } \partial \Omega .\end{cases}
$$

Let us choose $\delta>0$ small enough such that, if we set

$$
A_{\delta}=\left\{(\rho, \tau) \in A: \operatorname{dist}\left((\rho, \tau), \mathbb{R}^{2} \backslash A\right)>\delta\right\},
$$

then $\Sigma(\Omega) \cap A_{\delta} \neq \emptyset$ and

$$
\begin{aligned}
\inf \left\{\rho(x): x \in \Omega,\left(\rho(x), x_{n}\right) \in A_{\delta}\right\} & =\inf \left\{\rho(x): x \in \Omega,\left(\rho(x), x_{n}\right) \in A\right\} \\
& <\inf \left\{\rho(x): x \in \Omega,\left(\rho(x), x_{n}\right) \in \partial A_{\delta}\right\} .
\end{aligned}
$$

Then consider a smooth function $\theta: \mathbb{R}^{2} \rightarrow[0,1]$ such that $\theta(\rho, \tau)=1 \forall(\rho, \tau) \in A_{\delta}$ and $\theta(\rho, \tau)=0 \forall(\rho, \tau) \in$ $\mathbb{R}^{2} \backslash A$.

Let $g_{1}: \mathbb{R} \rightarrow \mathbb{R}^{+}$be the function defined by $g_{1}(t)=0 \forall t \leqslant 0, g_{1}(t)=t^{p-1} \forall t \geqslant 0$ and consider a smooth convex function $g_{0}: \mathbb{R} \rightarrow \mathbb{R}^{+}$such that $\lim _{t \rightarrow+\infty} g_{0}^{\prime}(t)<1, g_{0}(t) \leqslant g_{1}(t) \forall t \in \mathbb{R}$ and $g_{0}(t)=g_{1}(t) \forall t \leqslant t_{0}$ for a suitable $t_{0}>0$.

Then, let us consider the smooth function $g: \Omega \times \mathbb{R} \rightarrow \mathbb{R}$ defined by

$$
g(x, t)=\theta\left(\rho(x), x_{n}\right) g_{1}(t)+\left[1-\theta\left(\rho(x), x_{n}\right)\right] g_{0}(t)
$$

and the functional $f_{\varepsilon}: H_{S}(\Omega) \rightarrow \mathbb{R}$ defined by

$$
f_{\varepsilon}(u)=\frac{1}{2} \int_{\Omega}\left(\varepsilon^{2}|D u|^{2}+u^{2}\right) \mathrm{d} x-\int_{\Omega} G(x, u) \mathrm{d} x,
$$

where $G(x, t)=\int_{0}^{t} g(x, \tau) \mathrm{d} \tau$.

Throughout this paper, for every $E \subseteq \mathbb{R}^{2}$, we denote by $\widetilde{E}$ the set defined as follows

$$
\widetilde{E}=\left\{x=\left(x_{1}, \ldots, x_{n}\right) \in \mathbb{R}^{n}:\left(\rho(x), x_{n}\right) \in E\right\} .
$$

Notice that, since $\inf \{\rho(x): x \in \Omega \cap \tilde{A}\}>0$ because of condition (2.3), one can verify that $f_{\varepsilon}$ is well defined in $H_{S}(\Omega)$ and is a $C^{2}$-functional whose critical points (by the maximum principle) solve the problem

$$
\begin{cases}-\varepsilon^{2} \Delta u+u=g(x, u) & \text { in } \Omega, \\ u>0 & \text { in } \Omega, \\ u=0 & \text { on } \partial \Omega .\end{cases}
$$

It is clear that all nontrivial critical points for $f_{\varepsilon}$ belong to the set

$$
M_{\varepsilon}=\left\{u \in H_{S}(\Omega): u \not \equiv 0, f_{\varepsilon}^{\prime}(u)[u]=0\right\} .
$$


The following lemma describes some properties of $f_{\varepsilon}$ and $M_{\varepsilon}$ and, in particular, shows that looking for nontrivial critical points for $f_{\varepsilon}$ is equivalent to searching critical points for $f_{\varepsilon}$ constrained on $M_{\varepsilon}$.

Lemma 3.1. For all $p>2$ and $\varepsilon>0$, the following properties hold for the functional $f_{\varepsilon}$.

(a) Assume $u \in H_{S}(\Omega), u \not \equiv 0$; then, either $f_{\varepsilon}^{\prime}(t u)[u]>0 \forall t>0$ (what happens, for example, if $u^{+} \equiv 0$ in $\Omega \cap \tilde{A}$ ) or there exists a unique $t_{\varepsilon}>0$ such that $t_{\varepsilon} u \in M_{\varepsilon}$; in this case, $\left.f_{\varepsilon}^{\prime}(t u)[u]>0 \forall t \in\right] 0, t_{\varepsilon}\left[\right.$ and $f_{\varepsilon}^{\prime}(t u)[u]<0$ $\forall t>t_{\varepsilon}$ (this case occurs, for example, if $u^{+} \not \equiv 0$ in $\Omega \cap \tilde{A}_{\delta}$ ).

(b) $M_{\varepsilon} \neq \emptyset$ and $\inf _{M_{\varepsilon}} f_{\varepsilon}>0$.

(c) $M_{\varepsilon}$ is a $C^{1}$-manifold of codimension 1 ; moreover, every critical point for $f_{\varepsilon}$ constrained on $M_{\varepsilon}$ is a critical point for $f_{\varepsilon}$.

Proof. (a) First observe that, because of the definition of $g$, we have

$$
f_{\varepsilon}^{\prime}(t u)[u]=t \int_{\Omega}\left(\varepsilon^{2}|D u|^{2}+u^{2}\right) \mathrm{d} x>0 \quad \forall t>0
$$

for all $u$ such that $u^{+} \equiv 0$. If $u^{+} \not \equiv 0$, the function $t \mapsto \frac{1}{t} \int_{\Omega} g(x, t u) u \mathrm{~d} x$ is strictly increasing in $] 0,+\infty[$. Moreover, for all $t>0$, we have $g(x, t u) u=t^{p-1}\left(u^{+}\right)^{p}$ if $x \in \Omega \cap \tilde{A}_{\delta}$ while $0 \leqslant g(x, t u) u \leqslant\left[\lim _{s \rightarrow+\infty} g_{0}^{\prime}(s)\right] t u^{2}$ if $x \in \Omega \backslash \tilde{A}$.

Therefore, property (a) follows easily taking into account that

$$
f_{\varepsilon}^{\prime}(t u)[u]=t\left[\int_{\Omega}\left(\varepsilon^{2}|D u|^{2}+u^{2}\right) \mathrm{d} x-\frac{1}{t} \int_{\Omega} g(x, t u) u \mathrm{~d} x\right] \quad \forall t>0,
$$

where

$$
\begin{aligned}
& \lim _{t \rightarrow 0} \frac{1}{t} \int_{\Omega} g(x, t u) u \mathrm{~d} x=0, \\
& \frac{1}{t} \int_{\Omega \cap \tilde{A}_{\delta}} g(x, t u) u \mathrm{~d} x=t^{p-2} \int_{\Omega \cap \tilde{A}_{\delta}}\left(u^{+}\right)^{p} \mathrm{~d} x \quad \forall t>0
\end{aligned}
$$

and, since $\lim _{t \rightarrow+\infty} g_{0}^{\prime}(t)<1$,

$$
0 \leqslant \frac{1}{t} \int_{\Omega \backslash \tilde{A}} g(x, t u) u \mathrm{~d} x \leqslant \int_{\Omega \backslash \tilde{A}} u^{2} \mathrm{~d} x \quad \forall t>0 .
$$

(b) In order to prove that $M_{\varepsilon} \neq \emptyset$ for all $\varepsilon>0$ and $p>2$, it suffices to choose $\bar{\varphi} \in H_{S}(\Omega), \bar{\varphi} \geqslant 0$, such that $\bar{\varphi} \not \equiv 0$ in $\Omega \cap \tilde{A}_{\delta}$ (notice that $\Omega \cap \tilde{A}_{\delta} \neq \emptyset$ because of the choice of $\delta$ ). Then, from property (a) we infer that there exists $\bar{t}_{\varepsilon}>0$ such that $\bar{t}_{\varepsilon} \bar{\varphi} \in M_{\varepsilon}$. Moreover, property (a) implies that, for all $u \in M_{\varepsilon}$,

$$
f_{\varepsilon}(u) \geqslant f_{\varepsilon}(t u) \quad \forall t \geqslant 0 \text {. }
$$

On the other hand, we have $f_{\varepsilon}^{\prime}(0)=0$ and $f_{\varepsilon}^{\prime \prime}(0)[u, u]=\int_{\Omega}\left[\varepsilon^{2}|D u|^{2}+u^{2}\right] \mathrm{d} x$.

Therefore, there exists $r_{\varepsilon}>0$ and $\alpha_{\varepsilon}>0$ such that

$$
\inf \left\{f_{\varepsilon}(u): u \in H_{S}(\Omega), \int_{\Omega}|D u|^{2} \mathrm{~d} x=r_{\varepsilon}^{2}\right\} \geqslant \alpha_{\varepsilon} .
$$


It follows that

$$
\inf _{M_{\varepsilon}} f_{\varepsilon} \geqslant \alpha_{\varepsilon}>0
$$

which completes the proof of (b).

(c) The fact that $M_{\varepsilon}$ is a $C^{1}$ manifold of codimension 1 follows from the implicit function theorem. In fact, consider the functional $\Phi_{\varepsilon}: H_{S}(\Omega) \rightarrow \mathbb{R}$ defined by

$$
\Phi_{\varepsilon}(u)=f_{\varepsilon}^{\prime}(u)[u] \quad \forall u \in H_{S}(\Omega) .
$$

Taking into account condition (2.3), one can verify that $\Phi_{\varepsilon}$ is a $C^{1}$ functional. Moreover, $\Phi_{\varepsilon}^{\prime}(u) \neq 0$ for all $u \in M_{\varepsilon}$. In fact, if $u \in M_{\varepsilon}$, then $u^{+} \not \equiv 0$ and $\Phi_{\varepsilon}(u)=0$ that is

$$
\varepsilon^{2} \int_{\Omega}|D u|^{2} \mathrm{~d} x+\int_{\Omega} u^{2} \mathrm{~d} x-\int_{\Omega} g(x, u) u \mathrm{~d} x=0 .
$$

Therefore, if $g^{\prime}(x, t)$ denotes the derivative of $g(x, t)$ with respect to $t$, we have

$$
\begin{aligned}
\Phi_{\varepsilon}^{\prime}(u)[u] & =2 \varepsilon^{2} \int_{\Omega}|D u|^{2} \mathrm{~d} x+2 \int_{\Omega} u^{2} \mathrm{~d} x-\int_{\Omega} g^{\prime}(x, u) u^{2} \mathrm{~d} x-\int_{\Omega} g(x, u) u \mathrm{~d} x \\
& =\int_{\Omega}\left[g(x, u) u-g^{\prime}(x, u) u^{2}\right] \mathrm{d} x .
\end{aligned}
$$

On the other hand, a direct computation shows that $g(x, t) t-g^{\prime}(x, t) t^{2}<0 \forall t>0$. Therefore, since $u^{+} \not \equiv 0$, it follows that $\Phi_{\varepsilon}^{\prime}(u)[u]<0$. So $\Phi_{\varepsilon}^{\prime}(u) \neq 0$ and the implicit function theorem applies.

Now, let $u \in M_{\varepsilon}$ be a critical point for $f_{\varepsilon}$ constrained on $M_{\varepsilon}$. Then, there exists a Lagrange multiplier $\mu$ such that

$$
f_{\varepsilon}^{\prime}(u)+\mu \Phi_{\varepsilon}^{\prime}(u)=0 .
$$

It follows, in particular, that

$$
f_{\varepsilon}^{\prime}(u)[u]+\mu \Phi_{\varepsilon}^{\prime}(u)[u]=0,
$$

which implies $\mu=0$ because $f_{\varepsilon}^{\prime}(u)[u]=0$ and $\Phi_{\varepsilon}^{\prime}(u)[u] \neq 0$. Thus, $u$ is a critical point for $f_{\varepsilon}$.

Lemma 3.2. Under the assumptions of Theorem 2.1, the following properties hold for the functional $f_{\varepsilon}$ and the manifold $M_{\varepsilon}$ :

(a) $f_{\varepsilon}(u) \geqslant\left(\frac{1}{2}-\frac{1}{p}\right)\left\{\varepsilon^{2} \int_{\Omega}|D u|^{2} \mathrm{~d} x+\left[1-g_{0}^{\prime}(\infty)\right] \int_{\Omega} u^{2} \mathrm{~d} x\right\} \quad \forall u \in M_{\mathcal{E}}$,

where $g_{0}^{\prime}(\infty)=\lim _{t \rightarrow+\infty} g_{0}^{\prime}(t)$;

(b) $\inf \left\{\int_{\Omega \cap \tilde{A}}\left(u^{+}\right)^{p} \mathrm{~d} x: u \in M_{\varepsilon}\right\}>0$.

Proof. In order to prove (a), let us observe that, for all $u \in M_{\varepsilon}$,

$$
\begin{aligned}
0 & =\int_{\Omega}\left(\varepsilon^{2}|D u|^{2}+u^{2}\right) \mathrm{d} x-\int_{\Omega} g(x, u) u \mathrm{~d} x \\
& =\int_{\Omega}\left(\varepsilon^{2}|D u|^{2}+u^{2}\right) \mathrm{d} x-\int_{\Omega} \tilde{\theta}(x)\left(u^{+}\right)^{p} \mathrm{~d} x-\int_{\Omega}[1-\tilde{\theta}(x)] g_{0}(u) u \mathrm{~d} x,
\end{aligned}
$$


where $\tilde{\theta}(x)=\theta\left(\rho(x), x_{n}\right) \forall x \in \mathbb{R}^{n}$.

Now, set $G_{0}(t)=\int_{0}^{t} g_{0}(\tau) \mathrm{d} \tau$ and observe that

$$
G_{0}(t) \leqslant \frac{1}{2} g_{0}(t) t \quad \forall t \in \mathbb{R}
$$

because $g_{0}$ is a convex function. As a consequence, we obtain

$$
\begin{aligned}
f_{\varepsilon}(u) & =\frac{1}{2} \int_{\Omega}\left(\varepsilon^{2}|D u|^{2}+u^{2}\right) \mathrm{d} x-\int_{\Omega} G(x, u) \mathrm{d} x \\
& \geqslant \frac{1}{2} \int_{\Omega}\left(\varepsilon^{2}|D u|^{2}+u^{2}\right) \mathrm{d} x-\frac{1}{p} \int_{\Omega} \tilde{\theta}(x)\left(u^{+}\right)^{p} \mathrm{~d} x-\frac{1}{2} \int_{\Omega}[1-\tilde{\theta}(x)] g_{0}(u) u \mathrm{~d} x .
\end{aligned}
$$

It follows that, for all $u \in M_{\varepsilon}$,

$$
\begin{aligned}
f_{\varepsilon}(u) & \geqslant\left(\frac{1}{2}-\frac{1}{p}\right)\left[\int_{\Omega}\left(\varepsilon^{2}|D u|^{2}+u^{2}\right) \mathrm{d} x-\int_{\Omega}[1-\tilde{\theta}(x)] g_{0}(u) u \mathrm{~d} x\right] \\
& \geqslant\left(\frac{1}{2}-\frac{1}{p}\right)\left[\int_{\Omega}\left(\varepsilon^{2}|D u|^{2}+u^{2}\right) \mathrm{d} x-\int_{\Omega} g_{0}(u) u \mathrm{~d} x\right] .
\end{aligned}
$$

Now observe that, since $g_{0}$ is convex, we have $g_{0}(t) t \leqslant g_{0}^{\prime}(\infty) t^{2} \forall t \in \mathbb{R}$, which implies

$$
\int_{\Omega} g_{0}(u) u \mathrm{~d} x \leqslant g_{0}^{\prime}(\infty) \int_{\Omega} u^{2} \mathrm{~d} x .
$$

Thus, (a) follows easily from (3.25) and (3.26).

For the proof of (b), let us first prove that

$$
\int_{\Omega \cap \tilde{A}}\left(u^{+}\right)^{p} \mathrm{~d} x>0 \quad \forall u \in M_{\varepsilon} .
$$

For all $u \in M_{\varepsilon}$ we have

$$
\begin{aligned}
\int_{\Omega \cap \tilde{A}}\left(u^{+}\right)^{p} \mathrm{~d} x & \geqslant \int_{\Omega \cap \tilde{A}} g(x, u) u \mathrm{~d} x=\int_{\Omega}\left(\varepsilon^{2}|D u|^{2}+u^{2}\right) \mathrm{d} x-\int_{\Omega \backslash \tilde{A}} g(x, u) u \mathrm{~d} x \\
& \geqslant \varepsilon^{2} \int_{\Omega}|D u|^{2} \mathrm{~d} x+\left[1-g_{0}^{\prime}(\infty)\right] \int_{\Omega} u^{2} \mathrm{~d} x,
\end{aligned}
$$

which implies (3.27) (because $g_{0}^{\prime}(\infty)<1$ and $u \not \equiv 0 \forall u \in M_{\varepsilon}$ ).

Now observe that, $\operatorname{since} \inf \{\rho(x): x \in \Omega \cap \tilde{A}\}>0$ (see condition (2.3)), the subspace of $H^{1,2}(\Omega \cap \tilde{A})$ consisting of the radial functions, is compactly embedded in $L^{p}$; in particular, for a suitable positive constant $\bar{c}_{p}$, we have

$$
\int_{\Omega \cap \tilde{A}}|D u|^{2} \mathrm{~d} x \geqslant \bar{c}_{p}\left(\int_{\Omega \cap \tilde{A}}|u|^{p} \mathrm{~d} x\right)^{2 / p} \quad \forall u \in H_{S}(\Omega) .
$$

Therefore, taking into account (3.28), we obtain

$$
\int_{\Omega \cap \tilde{A}}\left(u^{+}\right)^{p} \mathrm{~d} x \geqslant \varepsilon^{2} \int_{\Omega}|D u|^{2} \mathrm{~d} x \geqslant \varepsilon^{2} \int_{\Omega \cap \tilde{A}}\left|D u^{+}\right|^{2} \mathrm{~d} x \geqslant \varepsilon^{2} \bar{c}_{p}\left(\int_{\Omega \cap \tilde{A}}\left(u^{+}\right)^{p} \mathrm{~d} x\right)^{2 / p} \quad \forall u \in M_{\varepsilon} .
$$


Since $\int_{\Omega \cap \tilde{A}}\left(u^{+}\right)^{p} \mathrm{~d} x \neq 0$ (see (3.27)), it follows

$$
\int_{\Omega \cap \tilde{A}}\left(u^{+}\right)^{p} \mathrm{~d} x \geqslant\left(\varepsilon^{2} \bar{c}_{p}\right)^{p /(p-2)} \quad \forall u \in M_{\varepsilon},
$$

which implies property (b).

Lemma 3.3. The minimum $\min _{M_{\varepsilon}} f_{\varepsilon}$ is achieved for all $\varepsilon>0$. Moreover, every minimizing function $u_{\varepsilon}$ solves problem (3.7).

Proof. Let $\left(u_{i}\right)_{i}$ be a minimizing sequence for $f_{\varepsilon}$ on $M_{\varepsilon}$, that is $u_{i} \in M_{\varepsilon} \forall i \in \mathbb{N}$ and $\lim _{i \rightarrow \infty} f_{\varepsilon}\left(u_{i}\right)=\inf _{M_{\varepsilon}} f_{\varepsilon}$. From (a) of Lemma 3.2 we infer that the sequence $\left(u_{i}\right)_{i}$ is bounded in $H_{0}^{1,2}(\Omega)$. It follows that, up to a subsequence, it converges to a function $u \in H_{S}(\Omega)$ weakly in $H_{0}^{1,2}(\Omega)$ and in $L^{2}(\Omega)$. Taking into account that the subspace of $H^{1,2}(\Omega \cap \tilde{A})$ consisting of the radial functions is compactly embedded in $L^{p}$ (because of condition (2.3)), we obtain

$$
\begin{aligned}
& \lim _{i \rightarrow \infty} \int_{\Omega \cap \tilde{A}}\left(u_{i}^{+}\right)^{p} \mathrm{~d} x=\int_{\Omega \cap \tilde{A}}\left(u^{+}\right)^{p} \mathrm{~d} x, \\
& \lim _{i \rightarrow \infty} \int_{\Omega} g\left(x, u_{i}\right) u_{i} \mathrm{~d} x=\int_{\Omega} g(x, u) u \mathrm{~d} x
\end{aligned}
$$

and

$$
\lim _{i \rightarrow \infty} \int_{\Omega} G\left(x, u_{i}\right) \mathrm{d} x=\int_{\Omega} G(x, u) \mathrm{d} x .
$$

In particular, (3.34) implies

$$
\lim _{i \rightarrow \infty} f_{\varepsilon}\left(u_{i}\right) \geqslant f_{\varepsilon}(u) .
$$

Therefore, if we show that $u \in M_{\varepsilon}$, we can conclude that $u$ is a minimizing function for $f_{\varepsilon}$ constrained on $M_{\varepsilon}$.

Notice that $u \not \equiv 0$ because $\int_{\Omega \cap \tilde{A}}\left(u^{+}\right)^{p} \mathrm{~d} x>0$, as we can infer from (3.32) taking into account (b) of Lemma 3.2. Thus, it remains to prove that $f_{\varepsilon}^{\prime}(u)[u]=0$, which (because of (3.33)) is equivalent to showing that

$$
\lim _{i \rightarrow \infty} \int_{\Omega}\left|D u_{i}\right|^{2} \mathrm{~d} x=\int_{\Omega}|D u|^{2} \mathrm{~d} x .
$$

In order to prove (3.36), we argue by contradiction and assume that (up to a subsequence)

$$
\int_{\Omega}|D u|^{2} \mathrm{~d} x<\lim _{i \rightarrow \infty} \int_{\Omega}\left|D u_{i}\right|^{2} \mathrm{~d} x,
$$

which (because of (3.33)) implies $f_{\varepsilon}^{\prime}(u)[u]<0$. As a consequence, taking into account (a) of Lemma 3.1, there exists $t \in] 0,1\left[\right.$ such that $t u \in M_{\varepsilon}$. It follows that

$$
\begin{aligned}
f_{\varepsilon}(t u) & =\int_{\Omega}\left[\frac{1}{2} g(x, t u) t u-G(x, t u)\right] \mathrm{d} x<\int_{\Omega}\left[\frac{1}{2} g(x, u) u-G(x, u)\right] \mathrm{d} x \\
& =\lim _{i \rightarrow \infty} \int_{\Omega}\left[\frac{1}{2} g\left(x, u_{i}\right) u_{i}-G\left(x, u_{i}\right)\right] \mathrm{d} x=\lim _{i \rightarrow \infty} f_{\varepsilon}\left(u_{i}\right)=\inf _{M_{\varepsilon}} f_{\varepsilon},
\end{aligned}
$$


which is a contradiction (notice that here the strict inequality holds because $u^{+} \not \equiv 0$ ).

Then, we can conclude that $u \in M_{\varepsilon}$ and $f_{\varepsilon}(u)=\min _{M_{\varepsilon}} f_{\varepsilon}$.

Finally, notice that every minimizing function for $f_{\varepsilon}$ on $M_{\varepsilon}$ is, in particular, a critical point for $f_{\varepsilon}$ constrained on $M_{\varepsilon}$; hence, the conclusion follows taking into account (c) of Lemma 3.1.

For each domain $\Sigma$ in $\mathbb{R}^{2}$, let us set

$$
m_{\varepsilon}(\Sigma)=\inf \left\{\int_{\Sigma}\left(\varepsilon^{2}|D v|^{2}+v^{2}\right) \mathrm{d} \xi: v \in H_{0}^{1,2}(\Sigma), \int_{\Sigma}|v|^{p} \mathrm{~d} \xi=1\right\} .
$$

It is well known (see $[3,8,9,26]$ ) that, for $\Sigma=\mathbb{R}^{2}$, the minimum $m_{\varepsilon}\left(\mathbb{R}^{2}\right)$ is achieved by a positive function which is unique modulo translations, radially symmetric with respect to the origin, decreasing when the radial coordinate increases and decaying exponentially at infinity together with its derivatives.

Lemma 3.4. For each domain $\Sigma$ of $\mathbb{R}^{2}$, the following properties hold for all $\varepsilon>0$ and $p>2$ :

(a) $m_{\varepsilon}(\Sigma)=\varepsilon^{2(p-2) / p} m_{1}\left(\frac{1}{\varepsilon} \Sigma\right)$;

(b) $\lim _{\varepsilon \rightarrow 0} \varepsilon^{-2(p-2) / p_{m}} m_{\varepsilon}(\Sigma)=m_{1}\left(\mathbb{R}^{2}\right)$.

Proof. For each function $v \in H_{0}^{1,2}(\Sigma)$, consider the function $v_{\varepsilon}$ in $H_{0}^{1,2}\left(\frac{1}{\varepsilon} \Sigma\right)$ defined by

$$
v_{\varepsilon}(\xi)=v(\varepsilon \xi) \quad \forall \xi \in \frac{1}{\varepsilon} \Sigma .
$$

A direct computation shows that

$$
\int_{\Sigma}\left(\varepsilon^{2}|D v|^{2}+v^{2}\right) \mathrm{d} \xi=\varepsilon^{2}\left[\int_{\frac{1}{\varepsilon} \Sigma}\left(\left|D v_{\varepsilon}\right|^{2}+v_{\varepsilon}^{2}\right) \mathrm{d} \xi\right]
$$

and

$$
\int_{\Sigma}|v|^{p} \mathrm{~d} \xi=\varepsilon^{2} \int_{\frac{1}{\varepsilon} \Sigma}\left|v_{\varepsilon}\right|^{p} \mathrm{~d} \xi
$$

If $v \not \equiv 0$, set $\bar{v}=v /\|v\|_{L^{p}(\Sigma)}$ and $\bar{v}_{\varepsilon}=v_{\varepsilon} /\left\|v_{\varepsilon}\right\|_{L^{p}\left(\frac{1}{\varepsilon} \Sigma\right)}$. Then, the above computations show that

$$
\int_{\Sigma}\left(\varepsilon^{2}|D \bar{v}|^{2}+\bar{v}^{2}\right) \mathrm{d} \xi=\varepsilon^{2(p-2) / 2} \int_{\frac{1}{\varepsilon} \Sigma}\left(\left|D \bar{v}_{\varepsilon}\right|^{2}+\bar{v}_{\varepsilon}^{2}\right) \mathrm{d} \xi,
$$

which clearly implies (a).

Taking into account (a), the proof of (b) is equivalent to showing that

$$
\lim _{\varepsilon \rightarrow 0} m_{1}\left(\frac{1}{\varepsilon} \Sigma\right)=m_{1}\left(\mathbb{R}^{2}\right) \text {. }
$$

It is clear that $m_{1}\left(\frac{1}{\varepsilon} \Sigma\right) \geqslant m_{1}\left(\mathbb{R}^{2}\right)$ for all $\varepsilon>0$ because each function of $H_{0}^{1,2}\left(\frac{1}{\varepsilon} \Sigma\right)$ can be extended by the value zero in $\mathbb{R}^{2} \backslash \frac{1}{\varepsilon} \Sigma$. Then, it is sufficient to show that for all $\varepsilon>0$ there exists a function $\bar{w}_{\varepsilon} \in H_{0}^{1,2}\left(\frac{1}{\varepsilon} \Sigma\right)$, $\left\|\bar{w}_{\varepsilon}\right\|_{L^{p}\left(\frac{1}{\varepsilon} \Sigma\right)}=1$, such that

$$
\lim _{\varepsilon \rightarrow 0} \int_{\frac{1}{\varepsilon} \Sigma}\left(\left|D \bar{w}_{\varepsilon}\right|^{2}+\bar{w}_{\varepsilon}^{2}\right) \mathrm{d} \xi=m_{1}\left(\mathbb{R}^{2}\right) .
$$


Let $\bar{w} \in H^{1,2}\left(\mathbb{R}^{2}\right)$ be the positive function (see $[3,8,9,26]$ ) such that

$$
\sup _{\mathbb{R}^{2}} \bar{w}=\bar{w}(0), \quad \int_{\mathbb{R}^{2}}|\bar{w}|^{p} \mathrm{~d} \xi=1, \quad \int_{\mathbb{R}^{2}}\left(|D \bar{w}|^{2}+\bar{w}^{2}\right) \mathrm{d} \xi=m_{1}\left(\mathbb{R}^{2}\right) .
$$

Let us fix $\xi_{0} \in \Sigma$ and consider a cut-off function $\varphi \in C_{0}^{\infty}(\Sigma)$, such that $0 \leqslant \varphi(\xi) \leqslant 1 \forall \xi \in \Sigma$ and $\varphi(\xi)=1$ $\forall \xi \in B\left(\xi_{0}, r_{0}\right)$ for a suitable $r_{0}>0$.

Now set $w_{\varepsilon}(\xi)=\varphi(\varepsilon \xi) \bar{w}\left(\xi-\xi_{0} / \varepsilon\right) \forall \xi \in \frac{1}{\varepsilon} \Sigma$ and $\bar{w}_{\varepsilon}=w_{\varepsilon} /\left\|w_{\varepsilon}\right\|_{L^{p}\left(\frac{1}{\varepsilon} \Sigma\right)}$.

Then, taking into account that $\bar{w}$ and its derivatives decay exponentially at infinity, a direct computation shows that (3.45) holds for the function $\bar{w}_{\varepsilon}$ defined as above.

Lemma 3.5. For all $p>2$, we have

$$
\limsup _{\varepsilon \rightarrow 0} \varepsilon^{-2} \min _{M_{\varepsilon}} f_{\varepsilon}<+\infty .
$$

Proof. Clearly, it suffices to show that for all $\varepsilon>0$ there exists $\tilde{u}_{\varepsilon} \in M_{\varepsilon}$ such that

$$
\limsup _{\varepsilon \rightarrow 0} \varepsilon^{-2} f_{\varepsilon}\left(\tilde{u}_{\varepsilon}\right)<+\infty .
$$

Choose $\xi_{0} \in \Sigma(\Omega) \cap A_{\delta}$ and $r_{0}>0$ such that $B\left(\xi_{0}, r_{0}\right) \subset \Sigma(\Omega) \cap A_{\delta}$. Since $B\left(\xi_{0}, r_{0}\right)$ is a bounded domain of $\mathbb{R}^{2}$, for all $p>2$ there exists a minimizing function $v_{\varepsilon} \in H_{0}^{1,2}\left(B\left(\xi_{0}, r_{0}\right)\right), v_{\varepsilon} \geqslant 0$ in $B\left(\xi_{0}, r_{0}\right)$, such that $\int_{B\left(\xi_{0}, r_{0}\right)}\left|v_{\varepsilon}\right|^{p} \mathrm{~d} \xi=1$ and

$$
\int_{B\left(\xi_{0}, r_{0}\right)}\left(\varepsilon^{2}\left|D v_{\varepsilon}\right|^{2}+v_{\varepsilon}^{2}\right) \mathrm{d} \xi=m_{\varepsilon}\left(B\left(\xi_{0}, r_{0}\right)\right) .
$$

Let $u_{\varepsilon} \in H_{0}^{1,2}(\Omega)$ be the function defined by $u_{\varepsilon}(x)=v_{\varepsilon}\left(\rho(x), x_{n}\right)$ if $\left(\rho(x), x_{n}\right) \in B\left(\xi_{0}, r_{0}\right), u_{\varepsilon}(x)=0$ otherwise.

A simple computation shows that there exist two constants $c_{1}$ and $c_{2}$, depending only on $\xi_{0}$ and $r_{0}$, such that

$$
\int_{\Omega}\left(\varepsilon^{2}\left|D u_{\varepsilon}\right|^{2}+u_{\varepsilon}^{2}\right) \mathrm{d} x \leqslant c_{1} \int_{B\left(\xi_{0}, r_{0}\right)}\left(\varepsilon^{2}\left|D v_{\varepsilon}\right|^{2}+v_{\varepsilon}^{2}\right) \mathrm{d} \xi
$$

and

$$
\int_{\Omega}\left|u_{\varepsilon}\right|^{p} \mathrm{~d} x \geqslant c_{2} \int_{B\left(\xi_{0}, r_{0}\right)}\left|v_{\varepsilon}\right|^{p} \mathrm{~d} \xi=c_{2}
$$

moreover, because of condition (2.3), we can choose $c_{2}>0$.

It follows that, if we set $\bar{u}_{\varepsilon}=u_{\varepsilon} /\left\|u_{\varepsilon}\right\|_{L^{p}(\Omega)}$, we have

$$
\int_{\Omega}\left(\varepsilon^{2}\left|D \bar{u}_{\varepsilon}\right|^{2}+\bar{u}_{\varepsilon}^{2}\right) \mathrm{d} x \leqslant c_{3} m_{\varepsilon}\left(B\left(\xi_{0}, r_{0}\right)\right)
$$

for a suitable constant $c_{3}$ depending only on $\xi_{0}, r_{0}$ and $p$.

Taking into account that $g\left(x, t \bar{u}_{\varepsilon}(x)\right)=\left|t \bar{u}_{\varepsilon}(x)\right|^{p-1} \forall t>0$, we infer that there exists $\bar{t}_{\varepsilon}>0$ such that $\tilde{u}_{\varepsilon}=$ $\bar{t}_{\varepsilon} \bar{u}_{\varepsilon} \in M_{\varepsilon}$.

Moreover, a direct computation shows that

$$
f_{\varepsilon}\left(\tilde{u}_{\varepsilon}\right)=\left(\frac{1}{2}-\frac{1}{p}\right)\left[\int_{\Omega}\left(\varepsilon^{2}\left|D \bar{u}_{\varepsilon}\right|^{2}+\bar{u}_{\varepsilon}^{2}\right) \mathrm{d} x\right]^{p /(p-2)} .
$$


It follows that, for a suitable constant $c_{4}$ (depending only on $\xi_{0}, r_{0}, p$ )

$$
\inf _{M_{\varepsilon}} f_{\varepsilon} \leqslant c_{4}\left[m_{\varepsilon}\left(B\left(\xi_{0}, r_{0}\right)\right)\right]^{p /(p-2)} .
$$

Hence (3.47) follows easily, taking into account (b) of Lemma 3.4.

Corollary 3.6. For all $\varepsilon>0$, let $u_{\varepsilon}$ be a minimizing function for $f_{\varepsilon}$ constrained on $M_{\varepsilon}$. Then

$$
\limsup _{\varepsilon \rightarrow 0} \int_{\Omega}\left|D u_{\varepsilon}\right|^{2} \mathrm{~d} x<+\infty \quad \text { and } \quad \limsup _{\varepsilon \rightarrow 0} \varepsilon^{-2} \int_{\Omega} u_{\varepsilon}^{2} \mathrm{~d} x<+\infty
$$

(which, in particular, implies $u_{\varepsilon} \rightarrow 0$ in $L^{2}(\Omega)$ as $\varepsilon \rightarrow 0$ ).

The proof is a direct consequence of Lemma 3.5 and (a) of Lemma 3.2 (taking into account that $g_{0}^{\prime}(\infty)<1$ ).

Lemma 3.7. For all $\varepsilon>0$, let $u_{\varepsilon}$ be a critical point for $f_{\varepsilon}$ constrained on $M_{\varepsilon}$. Then

$$
\sup _{\Omega} u_{\varepsilon} \geqslant 1 \text {. }
$$

Proof. Arguing by contradiction, assume that, for some $\varepsilon>0$,

$$
\sup _{\Omega} u_{\varepsilon}<1 \text {. }
$$

Then, we have

$$
0<g\left(x, u_{\varepsilon}(x)\right)<u_{\varepsilon}(x) \quad \forall x \in \Omega
$$

because of the maximum principle and the definition of $g$.

Let $e_{1}$ be a function in $H_{0}^{1,2}(\Omega)$ such that $e_{1}>0$ in $\Omega$ and $\Delta e_{1}+\lambda_{1} e_{1}=0$, where $\lambda_{1}$ denotes the first eigenvalue of the Laplace operator $-\Delta$ in $H_{0}^{1,2}(\Omega)$. Since $u_{\varepsilon}$ solves problem (3.7), a direct computation shows that

$$
\int_{\Omega}\left[g\left(x, u_{\varepsilon}\right)-u_{\varepsilon}-\varepsilon^{2} \lambda_{1} u_{\varepsilon}\right] e_{1} \mathrm{~d} x=0
$$

which is a contradiction because of (3.58).

Lemma 3.8. For all $\varepsilon>0$, let $u_{\varepsilon}$ be a minimizing function for $f_{\varepsilon}$ constrained on $M_{\varepsilon}$. Then $u_{\varepsilon}$ is bounded in $\Omega$ and it is a smooth solution of problem (3.7).

Proof. First observe that, since $\int_{\Omega}\left|D u_{\varepsilon}\right|^{2} \mathrm{~d} x<+\infty$ and $\inf \{\rho(x): x \in \Omega \cap \tilde{A}\}>0$, the function $u_{\varepsilon}$ (which has radial symmetry with respect to the $x_{n}$-axis) belongs to $L^{q}(\Omega \cap \tilde{A})$ for all $q \geqslant 1$. In particular, for $q>(p-1) \frac{n}{2}$, it follows that the function $w_{\varepsilon}$ defined by

$$
w_{\varepsilon}(x)=\frac{1}{n(n-2) \omega_{n} \varepsilon^{2}} \int_{\Omega \cap \tilde{A}} \frac{u_{\varepsilon}^{p-1}(y)}{|x-y|^{n-2}} \mathrm{~d} y,
$$

where $\omega_{n}$ denotes the measure of the unit ball of $\mathbb{R}^{n}$, is a bounded function in $H_{S}(\Omega)$. Taking into account that $g_{0}(t) \leqslant t \forall t \geqslant 0$, we have $\Delta\left(w_{\varepsilon}-u_{\varepsilon}\right) \leqslant 0$ (in weak sense) in $\Omega$; since $w_{\varepsilon} \geqslant 0$ on $\partial \Omega$, it follows that $u_{\varepsilon} \leqslant w_{\varepsilon}$ in $\Omega$. Therefore, $u_{\varepsilon}$ is bounded too; thus, since $g(x, t)$ is a smooth function, it follows that $u_{\varepsilon}$ is a smooth solution of (3.7). 
Lemma 3.9. For all $\alpha \in] 0,1\left[\right.$ and $\varepsilon>0$, let $u_{\varepsilon}$ be a minimizing function for $f_{\varepsilon}$ constrained on $M_{\varepsilon}$ and consider the set

$$
\Sigma_{\varepsilon}^{\alpha}=\left\{(\rho, \tau) \in \Sigma(\Omega): u_{\varepsilon}(\rho, 0, \ldots, 0, \tau)>\alpha\right\} .
$$

Then

(a) $\left.\operatorname{meas}\left(\Sigma_{\varepsilon}^{\alpha}\right)>0, \forall \varepsilon>0, \forall \alpha \in\right] 0,1[$;

(b) $\left.\lim _{\varepsilon \rightarrow 0} \operatorname{meas}\left(\Sigma_{\varepsilon}^{\alpha}\right)=0, \forall \alpha \in\right] 0,1[$;

(c) for all $\varepsilon>0$ and $\alpha \in] 0,1\left[\right.$, there exist $c_{\alpha, \varepsilon}=\left(\rho_{\alpha, \varepsilon}, \tau_{\alpha, \varepsilon}\right) \in \Sigma(\Omega)$ and $r_{\alpha, \varepsilon}>0$ such that

$$
\left.\Sigma_{\varepsilon}^{\alpha} \subseteq B\left(c_{\alpha, \varepsilon}, r_{\alpha, \varepsilon}\right) \quad \text { and } \quad \lim _{\varepsilon \rightarrow 0} r_{\alpha, \varepsilon}=0 \quad \forall \alpha \in\right] 0,1[\text {. }
$$

Moreover, we have

$$
\lim _{\varepsilon \rightarrow 0} \operatorname{dist}\left(c_{\alpha, \varepsilon}, A\right)=0 .
$$

Proof. Property (a) is a direct consequence of Lemma 3.7, while (b) follows from Corollary 3.6 (because $u_{\varepsilon} \rightarrow 0$ in $L^{2}(\Omega)$ as $\varepsilon \rightarrow 0$ ).

Property (c) is a consequence of the fact that $f_{\varepsilon}\left(u_{\varepsilon}\right)=\min _{M_{\varepsilon}} f_{\varepsilon}$, which implies the existence of $c_{\alpha, \varepsilon} \in \Sigma(\Omega)$ and $r_{\alpha, \varepsilon}>0$ such that (3.61) holds. In fact, let us argue by contradiction and assume that, for some $\left.\alpha \in\right] 0,1[$, (3.61) does not hold for any choice of $c_{\alpha, \varepsilon}$ and $r_{\alpha, \varepsilon}$. Taking into account the definition of $g(x, t)$, we can fix $\bar{\alpha}>0$, small enough, such that $g(x, \alpha)-\alpha<0 \forall \alpha \in] 0, \bar{\alpha}\left[, \forall x \in \Omega\right.$. Since $\Sigma_{\alpha^{\prime}, \varepsilon} \subseteq \Sigma_{\alpha, \varepsilon}$ for $\alpha^{\prime}>\alpha$, it is clear that, in order to prove our assertion, it suffices to consider only the case $\alpha \in] 0, \bar{\alpha}[$.

Then, fix $\alpha \in] 0, \bar{\alpha}\left[\right.$, consider the set $A^{\theta}=\left\{(\rho, \tau) \in \mathbb{R}^{2}: \theta(\rho, \tau)>0\right\}$ and observe that $\Sigma_{\alpha, \varepsilon} \cap A^{\theta} \neq \emptyset \forall \varepsilon>0$; in fact, otherwise, we should have $g\left(x, u_{\varepsilon}(x)\right)-u_{\varepsilon}(x)<0$ a.e. in $\Omega$ (since $g_{0}(t)<t \forall t>0$ ) which is impossible (by the maximum principle) because $u_{\varepsilon}>0$.

If the diameter of $\Sigma_{\alpha, \varepsilon}$ does not tend to zero as $\varepsilon \rightarrow 0$ (as we are assuming by contradiction), then $\widetilde{\Sigma}_{\alpha, \varepsilon}$ (see (3.6)) has at least two connected components for $\varepsilon>0$ small enough.

In fact, assume that (up to a subsequence)

$$
\lim _{\varepsilon \rightarrow 0} \operatorname{diam}\left(\Sigma_{\alpha, \varepsilon}\right)>0
$$

and, arguing by contradiction, assume also that (up to a subsequence) $\widetilde{\Sigma}_{\alpha, \varepsilon}$ is connected for all $\varepsilon>0$.

From (3.63) and condition (2.3) we infer that there exists $d>0$ such that

$$
d<\min \left\{\frac{1}{2} \lim _{\varepsilon \rightarrow 0} \operatorname{diam}\left(\Sigma_{\alpha, \varepsilon}\right), \inf \{\rho:(\rho, \tau) \in \Sigma(\Omega) \cap A\}\right\} .
$$

Since $\Sigma_{\alpha, \varepsilon} \cap A^{\theta} \neq \emptyset \forall \varepsilon>0$ and $A^{\theta} \subset A$, we can choose a point $p_{\varepsilon}$ in $\Sigma_{\alpha, \varepsilon} \cap A \forall \varepsilon>0$; then, for $\varepsilon>0$ small enough, there exist $p_{\varepsilon}^{\prime} \in \Sigma_{\alpha, \varepsilon}$, such that $\operatorname{dist}\left(p_{\varepsilon}, p_{\varepsilon}^{\prime}\right)=d$, and a continuous path in $\Sigma_{\alpha, \varepsilon} \cap B\left(p_{\varepsilon}, d\right)$, joining $p_{\varepsilon}$ and $p_{\varepsilon}^{\prime}$ (in fact $\Sigma_{\alpha, \varepsilon}$ is open connected, just as $\widetilde{\Sigma}_{\alpha, \varepsilon}$, and cannot be contained in $B\left(p_{\varepsilon}, d\right)$ as $\left.\operatorname{diam}\left(\Sigma_{\alpha, \varepsilon}\right)>2 d\right)$.

Now observe that, because of (3.64), we can choose $\bar{\rho}>0$ such that

$$
2 \bar{\rho}+d<\inf \{\rho:(\rho, \tau) \in \Sigma(\Omega) \cap A\} .
$$

Then, consider a smooth function $\zeta: \mathbb{R} \rightarrow[0,1]$ such that $\zeta(t)=0 \forall t \leqslant \bar{\rho}, \zeta(t)=1 \forall t \geqslant 2 \bar{\rho}$ and set $\varphi_{\varepsilon}(\rho, \tau)=$ $\zeta(\rho) u_{\varepsilon}(\rho, 0, \ldots, 0, \tau) \forall(\rho, \tau) \in \Sigma(\Omega)$. It is clear that $\varphi_{\varepsilon}$ is a smooth function in $H_{0}^{1}(\Sigma(\Omega))$ and $\varphi_{\varepsilon}(\rho, \tau)=$ $u_{\varepsilon}(\rho, 0, \ldots, 0, \tau) \forall(\rho, \tau) \in \Sigma(\Omega) \cap B\left(p_{\varepsilon}, d\right)$.

Moreover, since $\bar{\rho}>0$, Corollary 3.6 implies that

$$
\limsup _{\varepsilon \rightarrow 0} \int_{\Sigma(\Omega)}\left|D \varphi_{\varepsilon}\right|^{2} \mathrm{~d} \rho \mathrm{d} \tau<+\infty \quad \text { and } \quad \limsup _{\varepsilon \rightarrow 0} \frac{1}{\varepsilon^{2}} \int_{\Sigma(\Omega)} \varphi_{\varepsilon}^{2} \mathrm{~d} \rho \mathrm{d} \tau<+\infty .
$$


Now, consider a ball $B$ in $\mathbb{R}^{2}$ with centre in $(0,0)$ and radius large enough so that $\Sigma(\Omega) \subset B$; set

$$
S_{d}=\left\{(\rho, \tau) \in \mathbb{R}^{2}: \rho=0,|\tau| \leqslant \frac{d}{2}\right\}
$$

(notice that $S_{d} \subset B$ ) and denote by $\bar{\varphi}_{\varepsilon}$ the function in $H_{0}^{1}(B)$ which realizes the following minimum (see, for example, [11])

$$
\min \left\{\int_{B}|D \varphi|^{2} \mathrm{~d} \rho \mathrm{d} \tau: \varphi \in H_{0}^{1}(B),\|\varphi\|_{L^{2}(B)}=\left\|\varphi_{\varepsilon}\right\|_{L^{2}(\Sigma(\Omega))}, \varphi \geqslant \alpha \text { on } S_{d} \text { in the sense of } H_{0}^{1}(B)\right\} .
$$

Since $\varphi_{\varepsilon} \geqslant \alpha$ in the sense of $H_{0}^{1}(B)$ on a continuous path in $B$, joining $p_{\varepsilon}$ and $p_{\varepsilon}^{\prime}$, and since $\operatorname{dist}\left(p_{\varepsilon}, p_{\varepsilon}^{\prime}\right)=d$, we have

$$
\int_{B}\left|D \bar{\varphi}_{\varepsilon}\right|^{2} \mathrm{~d} \rho \mathrm{d} \tau \leqslant \int_{\Sigma(\Omega)}\left|D \varphi_{\varepsilon}\right|^{2} \mathrm{~d} \rho \mathrm{d} \tau \quad \forall \varepsilon>0
$$

(symmetry reasons justify this minimality property of the segment $S_{d}$ with respect to all the continuous paths in $B$ joining two arbitrary points having distance $d$ ).

It follows that $\lim _{\sup } \rightarrow 0 \int_{B}\left|D \bar{\varphi}_{\varepsilon}\right|^{2} \mathrm{~d} \rho \mathrm{d} \tau<+\infty$ (because of (3.66)). On the other hand, $\bar{\varphi}_{\varepsilon} \rightarrow 0$ in $L^{2}(B)$ as $\varepsilon \rightarrow 0$, because $\varphi_{\varepsilon} \rightarrow 0$ in $L^{2}\left(\Sigma(\Omega)\right.$ ) (see (3.66) and (3.67)). Therefore, up to a subsequence, $\bar{\varphi}_{\varepsilon} \rightarrow 0$ as $\varepsilon \rightarrow 0$ weakly in $H_{0}^{1}(B)$. Now, applying a theorem of Banach and Saks (see, for example, [24] and [11]), we infer that there exists a sequence $\left(\varepsilon_{i}\right)_{i}, \varepsilon_{i} \rightarrow 0$ as $i \rightarrow+\infty$, such that $\frac{1}{k} \sum_{i=1}^{k} \bar{\varphi}_{\varepsilon_{i}} \rightarrow 0$ as $k \rightarrow \infty$ (strongly) in $H_{0}^{1}(\Omega)$.

Clearly, this gives a contradiction because $\frac{1}{\alpha k} \sum_{i=1}^{k} \bar{\varphi}_{\varepsilon_{i}} \geqslant 1$ on $S_{d}$ in the sense of $H_{0}^{1}(B)$ for all $k \in \mathbb{N}$ and

$$
\text { cap } S_{d}:=\min \left\{\int_{B}|D \varphi|^{2} \mathrm{~d} \rho \mathrm{d} \tau: \varphi \in H_{0}^{1}(B), \varphi \geqslant 1 \text { on } S_{d} \text { in the sense of } H_{0}^{1}(B)\right\}>0 \text {. }
$$

Therefore, for $\varepsilon>0$ small enough, $\widetilde{\Sigma}_{\alpha, \varepsilon}$ must have at least two connected components; as a consequence, for $\varepsilon>0$ small enough, there exist $v_{1, \varepsilon}$ and $v_{2, \varepsilon}$ in $H_{0}^{1}(\Omega), v_{1, \varepsilon} \geqslant 0, v_{2, \varepsilon} \geqslant 0, \int_{\Omega} v_{1, \varepsilon} \mathrm{d} x>0, \int_{\Omega} v_{2, \varepsilon} \mathrm{d} x>0$, $\int_{\Omega} v_{1, \varepsilon} v_{2, \varepsilon} \mathrm{d} x=0$, such that $u_{\varepsilon}=u_{\varepsilon} \wedge \alpha+v_{1, \varepsilon}+v_{2, \varepsilon}$.

Notice that, as $\Sigma_{\alpha, \varepsilon} \cap A^{\theta} \neq \emptyset \forall \varepsilon>0$, we must have

$$
\operatorname{meas}\left(\tilde{A}^{\theta} \cap \operatorname{supp} v_{1, \varepsilon}\right)>0
$$

or meas $\left(\tilde{A}^{\theta} \cap \operatorname{supp} v_{2, \varepsilon}\right)>0$. So we can assume that (3.68) holds for every $\varepsilon>0$.

Then, let us consider the function $\bar{u}_{\varepsilon}=u_{\varepsilon} \wedge \alpha+v_{1, \varepsilon}=u_{\varepsilon}-v_{2, \varepsilon}$. It is clear that $\bar{u}_{\varepsilon} \in H_{0}^{1}(\Omega)$; moreover, notice that

$$
f_{\varepsilon}^{\prime}\left(\bar{u}_{\varepsilon}\right)\left[\bar{u}_{\varepsilon}\right]>0
$$

in fact, $\bar{u}_{\varepsilon}>0$ in $\Omega, \varepsilon^{2} \Delta u_{\varepsilon}-u_{\varepsilon}+g\left(x, u_{\varepsilon}\right)=0$ in $\Omega$ (see Lemma 3.8), $g(x, \alpha)-\alpha<0 \forall x \in \Omega$ because $\left.\alpha \in\right] 0, \bar{\alpha}[$, $\bar{u}_{\varepsilon}=u_{\varepsilon}$ where $v_{2, \varepsilon}=0, \bar{u}_{\varepsilon}=\alpha<u_{\varepsilon}$ where $v_{2, \varepsilon}>0$ (indeed, $-\varepsilon^{2} \Delta \bar{u}_{\varepsilon}+\bar{u}_{\varepsilon}-g\left(x, \bar{u}_{\varepsilon}\right)$ is a nonnegative measure on $\Omega$ ).

Now, for every $t \in \mathbb{R}$, let us consider the function $u_{\varepsilon, t}=u_{\varepsilon} \wedge \alpha+t v_{1, \varepsilon}$ and observe that, since (3.68) holds for every $\varepsilon>0$, we have

$$
\lim _{t \rightarrow+\infty} f_{\varepsilon}^{\prime}\left(u_{\varepsilon, t}\right)\left[u_{\varepsilon, t}\right]=-\infty \quad \forall \varepsilon>0 .
$$

From (3.69) and (3.70) it follows that there exists $\bar{t}>1$ such that $f_{\varepsilon}^{\prime}\left(u_{\varepsilon, \bar{t}}\right)\left[u_{\varepsilon, \bar{t}}\right]=0$, that is $u_{\varepsilon, \bar{t}} \in M_{\varepsilon}$.

We shall prove that $f_{\varepsilon}\left(u_{\varepsilon, \bar{t}}\right)<f_{\varepsilon}\left(u_{\varepsilon}\right)$, which gives a contradiction because $f_{\varepsilon}\left(u_{\varepsilon}\right)=\min _{M_{\varepsilon}} f_{\varepsilon}$ and $u_{\varepsilon, \bar{t}} \in M_{\varepsilon}$.

First we prove that $f_{\varepsilon}\left(\bar{u}_{\varepsilon}\right)<f_{\varepsilon}\left(u_{\varepsilon}\right)$; in fact, for every $t \in[0,1]$, 


$$
\begin{aligned}
& \frac{\mathrm{d}}{\mathrm{d} t} f_{\varepsilon}\left(u_{\varepsilon}-t v_{2, \varepsilon}\right)=f_{\varepsilon}^{\prime}\left(u_{\varepsilon}-t v_{2, \varepsilon}\right)\left[-v_{2, \varepsilon}\right] \\
& \quad=-\int_{\Omega}\left[\varepsilon^{2} D\left(u_{\varepsilon}-t v_{2, \varepsilon}\right) D v_{2, \varepsilon}+\left(u_{\varepsilon}-t v_{2, \varepsilon}\right) v_{2, \varepsilon}-g\left(x, u_{\varepsilon}-t v_{2, \varepsilon}\right) v_{2, \varepsilon}\right] \mathrm{d} x
\end{aligned}
$$

for $t=0$, we have $f_{\varepsilon}^{\prime}\left(u_{\varepsilon}\right)\left[-v_{2, \varepsilon}\right]=0$ because of Lemma 3.8; for $t=1$, taking into account that $\bar{u}_{\varepsilon}=\alpha$ where $v_{2, \varepsilon} \neq 0$, we obtain

$$
f_{\varepsilon}^{\prime}\left(\bar{u}_{\varepsilon}\right)\left[-v_{2, \varepsilon}\right]=-\int_{\Omega}[\alpha-g(x, \alpha)] v_{2, \varepsilon} \mathrm{d} x<0
$$

because $\alpha<\bar{\alpha}$. Thus, since $g(x, \cdot)$ is convex, it follows that $f_{\varepsilon}^{\prime}\left(u_{\varepsilon}-t v_{2, \varepsilon}\right)\left[-v_{2, \varepsilon}\right]<0$ for every $\left.t \in\right] 0,1[$, which implies $f_{\varepsilon}\left(\bar{u}_{\varepsilon}\right)<f_{\varepsilon}\left(u_{\varepsilon}\right)$.

Now we prove that $f_{\varepsilon}\left(u_{\varepsilon, \bar{t}}\right)<f_{\varepsilon}\left(\bar{u}_{\varepsilon}\right)$; in fact

$$
\frac{\mathrm{d}}{\mathrm{d} t} f_{\varepsilon}\left(u_{\varepsilon, t}\right)=f_{\varepsilon}^{\prime}\left(u_{\varepsilon, t}\right)\left[v_{1, \varepsilon}\right]=\int_{\Omega}\left[\varepsilon^{2} D u_{\varepsilon, t} D v_{1, \varepsilon}+u_{\varepsilon, t} v_{1, \varepsilon}-g\left(x, u_{\varepsilon, t}\right) v_{1, \varepsilon}\right] \mathrm{d} x ;
$$

for $t=0$, since $u_{\varepsilon, 0}=\alpha$ where $v_{1, \varepsilon} \neq 0$, we obtain

$$
f_{\varepsilon}^{\prime}\left(u_{\varepsilon, 0}\right)\left[v_{1, \varepsilon}\right]=\int_{\Omega}[\alpha-g(x, \alpha)] v_{1, \varepsilon} \mathrm{d} x>0
$$

because $\alpha<\bar{\alpha}$; for $t=1$, since $u_{\varepsilon, 1}=u_{\varepsilon}$ where $v_{1, \varepsilon} \neq 0$, we have

$$
f_{\varepsilon}^{\prime}\left(u_{\varepsilon, 1}\right)\left[v_{1, \varepsilon}\right]=\int_{\Omega}\left[\varepsilon^{2} D u_{\varepsilon} D v_{1, \varepsilon}+u_{\varepsilon} v_{1, \varepsilon}-g\left(x, u_{\varepsilon}\right) v_{1, \varepsilon}\right] \mathrm{d} x=f_{\varepsilon}^{\prime}\left(u_{\varepsilon}\right)\left[v_{1, \varepsilon}\right]=0
$$

because of Lemma 3.8. Therefore, since $g(x, \cdot)$ is convex, it follows that $f_{\varepsilon}^{\prime}\left(u_{\varepsilon, t}\right)\left[v_{1, \varepsilon}\right]<0$ for every $t>1$, which implies $f_{\varepsilon}\left(u_{\varepsilon, \bar{t}}\right)<f_{\varepsilon}\left(u_{\varepsilon, 1}\right)$ because $\bar{t}>1$.

Thus, since $u_{\varepsilon, 1}=\bar{u}_{\varepsilon}$, we have $f_{\varepsilon}\left(u_{\varepsilon, \bar{t}}\right)<f_{\varepsilon}\left(\bar{u}_{\varepsilon}\right)<f_{\varepsilon}\left(u_{\varepsilon}\right)$, which is impossible.

In order to prove (3.62), let us argue by contradiction and assume that (up to a subsequence) $\lim _{\varepsilon \rightarrow 0} \operatorname{dist}\left(c_{\alpha, \varepsilon}, A\right.$ ) $>0$. Then, since $u_{\varepsilon}$ is a smooth solution of problem (3.7) (see Lemma 3.8), for $\varepsilon>0$ small enough there exists a maximum point $x_{\varepsilon}$ for $u_{\varepsilon}$, such that $\operatorname{dist}\left(x_{\varepsilon}, \tilde{A}\right)>0$ and $u_{\varepsilon}\left(x_{\varepsilon}\right) \geqslant 1$. It is clear that this gives a contradiction because $g\left(x_{\varepsilon}, t\right)=g_{0}(t)<t$ for all $t>0$; in particular, for $t=u_{\varepsilon}\left(x_{\varepsilon}\right)$, we obtain $\Delta u_{\varepsilon}\left(x_{\varepsilon}\right)>0$ which is impossible because $x_{\varepsilon}$ is a maximum point for $u_{\varepsilon}$.

Lemma 3.10. For all $\alpha \in] 0,1\left[\right.$ and $\varepsilon>0$, let $c_{\alpha, \varepsilon}=\left(\rho_{\alpha, \varepsilon}, \tau_{\alpha, \varepsilon}\right) \in \Sigma(\Omega)$ be as in Lemma 3.9. Then, we have

$$
\left.\lim _{\varepsilon \rightarrow 0} \rho_{\alpha, \varepsilon}=\inf \left\{\rho(x): x \in \Omega,\left(\rho(x), x_{n}\right) \in A\right\} \quad \forall \alpha \in\right] 0,1[\text {. }
$$

Proof. Let us argue by contradiction and assume that, for some $\bar{\alpha} \in$ ]0, 1[, (up to a subsequence) we have $\lim _{\varepsilon \rightarrow 0} c_{\bar{\alpha}, \varepsilon}=\bar{c}=(\bar{\rho}, \bar{\tau}) \in \bar{A} \cap \overline{\Sigma(\Omega)}$ with

$$
\bar{\rho}>\inf \left\{\rho(x): x \in \Omega,\left(\rho(x), x_{n}\right) \in A\right\} .
$$

Lemma 3.9 implies that, if we set

$$
v_{\varepsilon}(\rho, \tau)=u_{\varepsilon}(\rho, 0, \ldots, 0, \tau) \quad \forall(\rho, \tau) \in \Sigma(\Omega)
$$

then $v_{\varepsilon} \rightarrow 0$, as $\varepsilon \rightarrow 0$, uniformly in $\Sigma(\Omega) \backslash B(\bar{c}, \eta)$ for all $\eta>0$; in fact, since $\left.\lim _{\varepsilon \rightarrow 0} r_{\alpha, \varepsilon}=0 \forall \alpha \in\right] 0,1[$ and $\Sigma_{\alpha^{\prime}, \varepsilon} \subseteq \Sigma_{\alpha, \varepsilon}$ for $\alpha^{\prime}>\alpha$, we have $\lim _{\varepsilon \rightarrow 0} c_{\alpha, \varepsilon}=\bar{c}$ for every $\alpha \in$ ]0,1[; so, for every $\alpha \in$ ]0, 1[, we have $\Sigma_{\alpha, \varepsilon} \subseteq B(\bar{c}, \eta)$ for $\varepsilon>0$ small enough. 
Now observe that, for every $\eta \in] 0, \bar{\rho}[$, we have

$$
\begin{aligned}
\int_{\Omega}\left(\varepsilon^{2}\left|D u_{\varepsilon}\right|^{2}+u_{\varepsilon}^{2}\right) \mathrm{d} x & \geqslant \int_{\Omega \cap \widetilde{B}(\bar{c}, \eta)}\left(\varepsilon^{2}\left|D u_{\varepsilon}\right|^{2}+u_{\varepsilon}^{2}\right) \mathrm{d} x \\
& =(n-1) \omega_{n-1} \int_{\Sigma(\Omega) \cap B(\bar{c}, \eta)}\left(\varepsilon^{2}\left|D v_{\varepsilon}\right|^{2}+v_{\varepsilon}^{2}\right) \rho^{n-2} \mathrm{~d} \rho \mathrm{d} \tau \\
& \geqslant(n-1) \omega_{n-1}(\bar{\rho}-\eta)^{n-2} \int_{\Sigma(\Omega) \cap B(\bar{c}, \eta)}\left(\varepsilon^{2}\left|D v_{\varepsilon}\right|^{2}+v_{\varepsilon}^{2}\right) \mathrm{d} \rho \mathrm{d} \tau .
\end{aligned}
$$

Therefore, taking into account Corollary 3.6, we infer that

$$
\limsup _{\varepsilon \rightarrow 0} \varepsilon^{-2} \int_{\Sigma(\Omega) \cap B(\bar{c}, \eta)}\left(\varepsilon^{2}\left|D v_{\varepsilon}\right|^{2}+v_{\varepsilon}^{2}\right) \mathrm{d} \rho \mathrm{d} \tau<+\infty,
$$

that is (if we set $v_{\varepsilon}(\rho, \tau)=0 \forall(\rho, \tau) \in B(\bar{c}, \eta) \backslash \Sigma(\Omega)$ )

$$
\limsup _{\varepsilon \rightarrow 0} \int_{B(\bar{c} / \varepsilon, \eta / \varepsilon)}\left(\left|D w_{\varepsilon}\right|^{2}+w_{\varepsilon}^{2}\right) \mathrm{d} \rho \mathrm{d} \tau<+\infty,
$$

where $w_{\varepsilon}$ denotes the function defined by $w_{\varepsilon}(\rho, \tau)=v_{\varepsilon}(\varepsilon \rho, \varepsilon \tau)$.

For all $\varepsilon>0$, let $\left(\rho_{\varepsilon}^{\prime}, \tau_{\varepsilon}^{\prime}\right) \in \Sigma(\Omega)$ be a maximum point for $v_{\varepsilon}$ (i.e. $\left.v_{\varepsilon}\left(\rho_{\varepsilon}^{\prime}, \tau_{\varepsilon}^{\prime}\right)=\max _{\Sigma(\Omega)} v_{\varepsilon}\right)$ and consider the function $\bar{w}_{\varepsilon}$ defined by

$$
\bar{w}_{\varepsilon}(\rho, \tau)=w_{\varepsilon}\left(\rho+\rho_{\varepsilon}^{\prime} / \varepsilon, \tau+\tau_{\varepsilon}^{\prime} / \varepsilon\right)=v_{\varepsilon}\left(\varepsilon \rho+\rho_{\varepsilon}^{\prime}, \varepsilon \tau+\tau_{\varepsilon}^{\prime}\right) .
$$

Notice that Lemma 3.9 implies $\lim _{\varepsilon \rightarrow 0}\left(\rho_{\varepsilon}^{\prime}, \tau_{\varepsilon}^{\prime}\right)=\bar{c}$; therefore, for every bounded subset $\Sigma$ of $\mathbb{R}^{2}$, we have $\varepsilon \Sigma+$ $\left(\rho_{\varepsilon}^{\prime}, \tau_{\varepsilon}^{\prime}\right) \subseteq B(\bar{c}, \eta)$ that is

$$
\Sigma+\left(\rho_{\varepsilon}^{\prime} / \varepsilon, \tau_{\varepsilon}^{\prime} / \varepsilon\right) \subseteq B(\bar{c} / \varepsilon, \eta / \varepsilon)
$$

for $\varepsilon>0$ small enough.

It follows that, for all $\varepsilon>0$ small enough,

$$
\int_{\Sigma}\left(\left|D \bar{w}_{\varepsilon}\right|^{2}+\bar{w}_{\varepsilon}^{2}\right) \mathrm{d} \rho \mathrm{d} \tau \leqslant \int_{B(\bar{c} / \varepsilon, \eta / \varepsilon)}\left(\left|D w_{\varepsilon}\right|^{2}+w_{\varepsilon}^{2}\right) \mathrm{d} \rho \mathrm{d} \tau,
$$

which, because of (3.81), implies

$$
\limsup _{\varepsilon \rightarrow 0} \int_{\Sigma}\left(\left|D \bar{w}_{\varepsilon}\right|^{2}+\bar{w}_{\varepsilon}^{2}\right) \mathrm{d} \rho \mathrm{d} \tau<+\infty
$$

for every bounded domain $\Sigma$ of $\mathbb{R}^{2}$.

As a consequence, there exists $\bar{w} \in H^{1}\left(\mathbb{R}^{2}\right)$ such that (up to a subsequence) $\bar{w}_{\varepsilon} \rightarrow \bar{w}$ weakly in $H^{1}\left(\mathbb{R}^{2}\right)$, a.e. in $\mathbb{R}^{2}$ and in $L^{q}(\Sigma) \forall q \geqslant 1$ for every bounded domain $\Sigma$ of $\mathbb{R}^{2}$.

Now observe that the function $\hat{u}_{\varepsilon}$, defined by $\hat{u}_{\varepsilon}(x)=u_{\varepsilon}\left(\varepsilon x+x_{\varepsilon}^{\prime}\right)$, with $x_{\varepsilon}^{\prime}=\left(\rho_{\varepsilon}^{\prime}, 0, \ldots, 0, \tau_{\varepsilon}^{\prime}\right)$, solves the following problem

$$
\begin{cases}-\Delta \hat{u}_{\varepsilon}+\hat{u}_{\varepsilon}=g\left(\varepsilon x+x_{\varepsilon}^{\prime}, \hat{u}_{\varepsilon}\right) & \text { in } \Omega_{\varepsilon}, \\ \hat{u}_{\varepsilon}>0 & \text { in } \Omega_{\varepsilon}, \\ \hat{u}_{\varepsilon}=0 & \text { on } \partial \Omega_{\varepsilon},\end{cases}
$$


where $\Omega_{\varepsilon}=\frac{1}{\varepsilon}\left(\Omega-x_{\varepsilon}^{\prime}\right)$. Therefore, taking also into account that $\lim _{\varepsilon \rightarrow 0} \rho_{\varepsilon}^{\prime}=\bar{\rho}>0$ and that $\hat{u}_{\varepsilon}(0)=\bar{w}_{\varepsilon}(0)=$ $\max _{\Omega} u_{\varepsilon} \geqslant 1$, we can say that, in its support, $\bar{w}$ solves the equation

$$
-\Delta \bar{w}+\bar{w}=g(\bar{x}, \bar{w})
$$

where $\bar{x}=(\bar{\rho}, 0, \ldots, 0, \bar{\tau})$, and that $\bar{w} \not \equiv 0$ (in fact, if $\bar{w} \equiv 0$, since $\bar{w}_{\varepsilon} \rightarrow \bar{w}$ in $L^{q}(\Sigma) \forall q \geqslant 1$ for every bounded domain $\Sigma$ of $\mathbb{R}^{2}$ and $\hat{u}_{\varepsilon}$ solves problem (3.85), we could infer that $\bar{w}_{\varepsilon}(0) \rightarrow 0$, which is a contradiction).

Now, consider the functional $\bar{f}: H^{1}\left(\mathbb{R}^{2}\right) \rightarrow \mathbb{R}$ defined by

$$
\bar{f}(w)=\frac{1}{2} \int_{\mathbb{R}^{2}}\left(|D w|^{2}+w^{2}\right) \mathrm{d} \rho \mathrm{d} \tau-\int_{\mathbb{R}^{2}} G(\bar{x}, w) \mathrm{d} \rho \mathrm{d} \tau
$$

and observe that, since $\bar{f}(\bar{w})=\max _{t>0} \bar{f}(t \bar{w})$ and $G(\bar{x}, t) \leqslant \frac{1}{p}|t|^{p} \forall t \in \mathbb{R}$, then $\bar{f}(\bar{w}) \geqslant \max _{t>0} \hat{f}(t \bar{w})$, where $\hat{f}$ is the functional defined in $H^{1}\left(\mathbb{R}^{2}\right)$ by

$$
\hat{f}(w)=\frac{1}{2} \int_{\mathbb{R}^{2}}\left(|D w|^{2}+w^{2}\right) \mathrm{d} \rho \mathrm{d} \tau-\frac{1}{p} \int_{\mathbb{R}^{2}}|w|^{p} \mathrm{~d} \rho \mathrm{d} \tau .
$$

On the other hand, we have

$$
\int_{\mathbb{R}^{2}}\left(|D \bar{w}|^{2}+\bar{w}^{2}\right) \mathrm{d} \rho \mathrm{d} \tau \geqslant m_{1}\left(\mathbb{R}^{2}\right)\left(\int_{\mathbb{R}^{2}}|\bar{w}|^{p}\right)^{2 / p} ;
$$

thus, we obtain

$$
\bar{f}(\bar{w}) \geqslant \max _{t>0}\left\{\frac{1}{2} m_{1}\left(\mathbb{R}^{2}\right) t^{2}\|\bar{w}\|_{L^{p}\left(\mathbb{R}^{2}\right)}^{2}-\frac{t^{p}}{p}\|\bar{w}\|_{L^{p}\left(\mathbb{R}^{2}\right)}^{p}\right\}=\left(\frac{1}{2}-\frac{1}{p}\right)\left[m_{1}\left(\mathbb{R}^{2}\right)\right]^{p /(p-2)} .
$$

Now observe that, since $u_{\varepsilon} \in M_{\varepsilon}$ and $\frac{1}{2} g(x, t) t-G(x, t) \geqslant 0 \forall x \in \Omega, \forall t \in \mathbb{R}$, we have

$$
\begin{aligned}
f_{\varepsilon}\left(u_{\varepsilon}\right) & =\int_{\Omega}\left[\frac{1}{2} g\left(x, u_{\varepsilon}\right) u_{\varepsilon}-G\left(x, u_{\varepsilon}\right)\right] \mathrm{d} x \\
& \geqslant \int_{\Omega \cap \widetilde{B}(\bar{c}, \eta)}\left[\frac{1}{2} g\left(x, u_{\varepsilon}\right) u_{\varepsilon}-G\left(x, u_{\varepsilon}\right)\right] \mathrm{d} x \\
& \geqslant(n-1) \omega_{n-1}(\bar{\rho}-\eta)^{n-2} \int_{\Sigma(\Omega) \cap B(\bar{c}, \eta)}\left[\frac{1}{2} g\left(\rho, 0, \ldots, 0, \tau, v_{\varepsilon}\right) v_{\varepsilon}-G\left(\rho, 0, \ldots, 0, \tau, v_{\varepsilon}\right)\right] \mathrm{d} \rho \mathrm{d} \tau .
\end{aligned}
$$

It follows that

$$
\begin{aligned}
\liminf _{\varepsilon \rightarrow 0} \varepsilon^{-2} f_{\varepsilon}\left(u_{\varepsilon}\right) \geqslant & (n-1) \omega_{n-1}(\bar{\rho}-\eta)^{n-2} \liminf _{\varepsilon \rightarrow 0} \int_{\Sigma(\Omega) \cap B(\bar{c}, \eta)}\left[\frac{1}{2} g\left(\rho, 0, \ldots, 0, \tau, v_{\varepsilon}\right) v_{\varepsilon}\right. \\
& \left.-G\left(\rho, 0, \ldots, 0, \tau, v_{\varepsilon}\right)\right] \mathrm{d} \rho \mathrm{d} \tau
\end{aligned}
$$

that is (since we set $v_{\varepsilon}=0$ in $B(\bar{c}, \eta) \backslash \Sigma(\Omega)$ ) 


$$
\begin{aligned}
\liminf _{\varepsilon \rightarrow 0} \varepsilon^{-2} f_{\varepsilon}\left(u_{\varepsilon}\right) \geqslant & (n-1) \omega_{n-1}(\bar{\rho}-\eta)^{n-2} \liminf _{\varepsilon \rightarrow 0} \int_{B(\bar{c} / \varepsilon, \eta / \varepsilon)}\left[\frac{1}{2} g\left(\varepsilon \rho, 0, \ldots, 0, \varepsilon \tau, w_{\varepsilon}\right) w_{\varepsilon}\right. \\
& \left.-G\left(\varepsilon \rho, 0, \ldots, 0, \varepsilon \tau, w_{\varepsilon}\right)\right] \mathrm{d} \rho \mathrm{d} \tau .
\end{aligned}
$$

Taking into account (3.82), for every bounded domain $\Sigma$ of $\mathbb{R}^{2}$ we have

$$
\begin{aligned}
\liminf _{\varepsilon \rightarrow 0} \varepsilon^{-2} f_{\varepsilon}\left(u_{\varepsilon}\right) \geqslant & (n-1) \omega_{n-1}(\bar{\rho}-\eta)^{n-2} \liminf _{\varepsilon \rightarrow 0} \int_{\Sigma}\left[\frac{1}{2} g\left(\varepsilon \rho+\rho_{\varepsilon}^{\prime}, 0, \ldots, 0, \varepsilon \tau+\tau_{\varepsilon}^{\prime}, \bar{w}_{\varepsilon}\right) \bar{w}_{\varepsilon}\right. \\
& \left.-G\left(\varepsilon \rho+\rho_{\varepsilon}^{\prime}, 0, \ldots, 0, \varepsilon \tau+\tau_{\varepsilon}^{\prime}, \bar{w}_{\varepsilon}\right)\right] \mathrm{d} \rho \mathrm{d} \tau .
\end{aligned}
$$

Since $\bar{w}_{\varepsilon} \rightarrow \bar{w}$ in $L^{p}(\Sigma)$, we obtain

$$
\liminf _{\varepsilon \rightarrow 0} \varepsilon^{-2} f_{\varepsilon}\left(u_{\varepsilon}\right) \geqslant(n-1) \omega_{n-1}(\bar{\rho}-\eta)^{n-2} \int_{\Sigma}\left[\frac{1}{2} g(\bar{x}, \bar{w}) \bar{w}-G(\bar{x}, \bar{w})\right] \mathrm{d} \rho \mathrm{d} \tau
$$

for every bounded domain $\Sigma$ in $\mathbb{R}^{2}$, which implies

$$
\liminf _{\varepsilon \rightarrow 0} \varepsilon^{-2} f_{\varepsilon}\left(u_{\varepsilon}\right) \geqslant(n-1) \omega_{n-1}(\bar{\rho}-\eta)^{n-2} \int_{\mathbb{R}^{2}}\left[\frac{1}{2} g(\bar{x}, \bar{w}) \bar{w}-G(\bar{x}, \bar{w})\right] \mathrm{d} \rho \mathrm{d} \tau .
$$

On the other hand, we have

$$
\int_{\mathbb{R}^{2}}\left[\frac{1}{2} g(\bar{x}, \bar{w}) \bar{w}-G(\bar{x}, \bar{w})\right] \mathrm{d} \rho \mathrm{d} \tau=\bar{f}(\bar{w}),
$$

because $\int_{\mathbb{R}^{2}} g(\bar{x}, \bar{w}) \bar{w} \mathrm{~d} \rho \mathrm{d} \tau=\int_{\mathbb{R}^{2}}\left(|D \bar{w}|^{2}+\bar{w}^{2}\right) \mathrm{d} \rho \mathrm{d} \tau$.

Thus, by (3.89), we get

$$
\left.\liminf _{\varepsilon \rightarrow 0} \varepsilon^{-2} f_{\varepsilon}\left(u_{\varepsilon}\right) \geqslant\left(\frac{1}{2}-\frac{1}{p}\right)(n-1) \omega_{n-1}(\bar{\rho}-\eta)^{n-2}\left[m_{1}\left(\mathbb{R}^{2}\right)\right]^{p /(p-2)} \quad \forall \eta \in\right] 0, \bar{\rho}[
$$

and, letting $\eta \rightarrow 0$,

$$
\liminf _{\varepsilon \rightarrow 0} \varepsilon^{-2} f_{\varepsilon}\left(u_{\varepsilon}\right) \geqslant\left(\frac{1}{2}-\frac{1}{p}\right)(n-1) \omega_{n-1} \bar{\rho}^{n-2}\left[m_{1}\left(\mathbb{R}^{2}\right)\right]^{p /(p-2)} .
$$

In order to prove that (3.98) gives a contradiction, observe that, since $\bar{\rho}>\inf \left\{\rho(x): x \in \Omega,\left(\rho(x), x_{n}\right) \in A\right\}$, because of the choice of $\delta$ (see (3.3)) there exists $\bar{c}_{0}=\left(\bar{\rho}_{0}, \bar{\tau}_{0}\right) \in A_{\delta} \cap \Sigma(\Omega)$ such that $\bar{\rho}_{0}<\bar{\rho}$. Fix $\bar{\eta}>0$ small enough so that $B\left(\bar{c}_{0}, \bar{\eta}\right) \subset A_{\delta} \cap \Sigma(\Omega)$; then consider a function $\bar{v}_{\varepsilon} \in H_{0}^{1,2}\left(B\left(\bar{c}_{0}, \bar{\eta}\right)\right), \bar{v}_{\varepsilon}>0$ in $B\left(\bar{c}_{0}, \bar{\eta}\right)$, such that

$$
\int_{B\left(\overline{c_{0}}, \bar{\eta}\right)} \bar{v}_{\varepsilon}^{p} \mathrm{~d} \rho \mathrm{d} \tau=1 \quad \text { and } \int_{B\left(\bar{c}_{0}, \bar{\eta}\right)}\left(\varepsilon^{2}\left|D \bar{v}_{\varepsilon}\right|^{2}+\bar{v}_{\varepsilon}^{2}\right) \mathrm{d} \rho \mathrm{d} \tau=m_{\varepsilon}\left(B\left(\bar{c}_{0}, \bar{\eta}\right)\right) .
$$

Let $\bar{u}_{\varepsilon}$ be the function in $H_{S}(\Omega)$ defined by $\bar{u}_{\varepsilon}(x)=\bar{v}_{\varepsilon}\left(\rho(x), x_{n}\right)$ if $\left(\rho(x), x_{n}\right) \in B\left(\bar{c}_{0}, \bar{\eta}\right), \bar{u}_{\varepsilon}(x)=0$ otherwise; notice that, since $B\left(\bar{c}_{0}, \bar{\eta}\right) \subseteq A_{\delta}$, there exists $\bar{t}_{\varepsilon}>0$ such that $\bar{t}_{\varepsilon} \bar{u}_{\varepsilon} \in M_{\varepsilon}$. Moreover, a direct computation shows that

$$
f_{\varepsilon}\left(\bar{t}_{\varepsilon} \bar{u}_{\varepsilon}\right)=\left(\frac{1}{2}-\frac{1}{p}\right)\left[\left(\int_{\Omega} \bar{u}_{\varepsilon}^{p} \mathrm{~d} x\right)^{-2 / p} \int_{\Omega}\left(\varepsilon^{2}\left|D \bar{u}_{\varepsilon}\right|^{2}+\bar{u}_{\varepsilon}^{2}\right) \mathrm{d} x\right]^{p /(p-2)} .
$$


Now, observe that

$$
\begin{aligned}
\int_{\Omega}\left(\varepsilon^{2}\left|D \bar{u}_{\varepsilon}\right|^{2}+\bar{u}_{\varepsilon}^{2}\right) \mathrm{d} x & \leqslant(n-1) \omega_{n-1}\left(\bar{\rho}_{0}+\bar{\eta}\right)^{n-2} \int_{B\left(\bar{c}_{0}, \bar{\eta}\right)}\left(\varepsilon^{2}\left|D \bar{v}_{\varepsilon}\right|^{2}+\bar{v}_{\varepsilon}^{2}\right) \mathrm{d} \rho \mathrm{d} \tau \\
& =(n-1) \omega_{n-1}\left(\bar{\rho}_{0}+\bar{\eta}\right)^{n-2} m_{\varepsilon}\left(B\left(\bar{c}_{0}, \bar{\eta}\right)\right)
\end{aligned}
$$

and

$$
\int_{\Omega} \bar{u}_{\varepsilon}^{p} \mathrm{~d} x \geqslant(n-1) \omega_{n-1}\left(\bar{\rho}_{0}-\bar{\eta}\right)^{n-2} .
$$

It follows that

$$
f_{\varepsilon}\left(\bar{t}_{\varepsilon} \bar{u}_{\varepsilon}\right) \leqslant\left(\frac{1}{2}-\frac{1}{p}\right)(n-1) \omega_{n-1}\left(\bar{\rho}_{0}+\bar{\eta}\right)^{(n-2) p /(p-2)}\left(\bar{\rho}_{0}-\bar{\eta}\right)^{-2(n-2) /(p-2)}\left[m_{\varepsilon}\left(B\left(\bar{c}_{0}, \bar{\eta}\right)\right)\right]^{p /(p-2)},
$$

which, because of Lemma 3.4, implies

$$
\limsup _{\varepsilon \rightarrow 0} \varepsilon^{-2} f_{\varepsilon}\left(\bar{t}_{\varepsilon} \bar{u}_{\varepsilon}\right) \leqslant\left(\frac{1}{2}-\frac{1}{p}\right)(n-1) \omega_{n-1}\left(\bar{\rho}_{0}+\bar{\eta}\right)^{(n-2) p /(p-2)}\left(\bar{\rho}_{0}-\bar{\eta}\right)^{-2(n-2) /(p-2)}\left[m_{1}\left(\mathbb{R}^{2}\right)\right]^{p /(p-2)} .
$$

Notice that, since $\bar{\rho}_{0}<\bar{\rho}$, we can choose $\bar{\eta}$ small enough so that, in addition, it satisfies the condition

$$
\left(\bar{\rho}_{0}+\bar{\eta}\right)^{(n-2) p /(p-2)}\left(\bar{\rho}_{0}-\bar{\eta}\right)^{-2(n-2) /(p-2)}<\bar{\rho}^{n-2} .
$$

Thus, from (3.98) and (3.105) we infer that $f_{\varepsilon}\left(\bar{t}_{\varepsilon} \bar{u}_{\varepsilon}\right)<f_{\varepsilon}\left(u_{\varepsilon}\right)$ for $\varepsilon>0$ small enough. It is clear that it is a contradiction because $f_{\varepsilon}\left(u_{\varepsilon}\right)=\min _{M_{\varepsilon}} f_{\varepsilon}$ and $\bar{t}_{\varepsilon} \bar{u}_{\varepsilon} \in M_{\varepsilon}$.

Proof of Theorem 2.1. Let $u_{\varepsilon}$ be a minimizing function for $f_{\varepsilon}$ constrained on $M_{\varepsilon}$ (which there exists for all $\varepsilon>0$ because of Lemma 3.3). Since $u_{\varepsilon}$ is a solution of problem (3.7), in order to prove that it solves also problem (3.1), it suffices to observe that, for $\varepsilon$ small enough, $g\left(x, u_{\varepsilon}(x)\right)=\left[u_{\varepsilon}(x)\right]^{p-1}$ for all $x \in \Omega$, which follows easily from Lemmas 3.9 and 3.10, since they imply that

$$
\lim _{\varepsilon \rightarrow 0} \sup \left\{u_{\varepsilon}(x): x \in \Omega,\left(\rho(x), x_{n}\right) \notin A_{\delta}\right\}=0 .
$$

Therefore, since the solvability of problem (1.1) for large $\lambda$ is equivalent to the solvability of (3.1) for small $\varepsilon$, the existence of a solution of (1.1) for large $\lambda$ is proved.

Lemma 3.8 guarantees that the solution is bounded and smooth in $\Omega$.

Property (2.4) is a direct consequence of Lemma 3.9, while (2.5) is proved in Lemma 3.7. The asymptotic behaviour of $c_{\lambda}$, as $\lambda \rightarrow+\infty$, follows from Lemmas 3.9 and 3.10 .

Finally, Corollary 3.6 guarantees (2.7), (2.8) and (taking into account that $u_{\varepsilon} \in M_{\varepsilon}$ ) also (2.9).

Remark 3.11. The rescaling arguments used in the proof of Theorem 2.1 and the concentration-compactness principle (see [10]) can be also used to obtain information on the asymptotic shape of the solution $u_{\lambda}$ as $\lambda \rightarrow+\infty$. In particular, using this methods, one can show that there exists $\left(\tilde{\rho}_{\lambda}, \tilde{\tau}_{\lambda}\right) \in \mathbb{R}^{2}$ (for all $\lambda \geqslant \bar{\lambda}$ ) such that the function

$$
v_{\lambda}(\rho, \tau)=\lambda^{-1 /(p-2)} u_{\lambda}\left(\frac{\rho-\tilde{\rho}_{\lambda}}{\sqrt{\lambda}}, 0, \ldots, 0, \frac{\tau-\tilde{\tau}_{\lambda}}{\sqrt{\lambda}}\right)
$$

converges, as $\lambda \rightarrow+\infty$, to the solution of problem (1.2). 
Remark 3.12. Notice that solutions of problem (3.1) can be also obtained as critical points for the functional $F_{\varepsilon}$ defined by

$$
F_{\varepsilon}(u)=\frac{1}{2} \int_{\Omega}\left[\varepsilon^{2}|D u|^{2}+u^{2}\right] \mathrm{d} x-\frac{1}{p} \int_{\Omega}\left(u^{+}\right)^{p} \mathrm{~d} x,
$$

constrained on the manifold

$$
V_{\varepsilon}=\left\{u \in H_{S}(\Omega): u \neq \equiv, \int_{\Omega}\left(\varepsilon^{2}|D u|^{2}+u^{2}\right) \mathrm{d} x=\int_{\Omega}\left(u^{+}\right)^{p} \mathrm{~d} x\right\} .
$$

In particular, one can try to minimize $F_{\varepsilon}$ on $V_{\varepsilon}$. But, if $\Omega$ meets the $x_{n}$-axis, this approach gives solutions of different nature when $p<\frac{2 n}{n-2}$ and does not work when $p \geqslant \frac{2 n}{n-2}$. In fact, if $p<\frac{2 n}{n-2}$, one can show that the minimizing functions for $f_{\varepsilon}$ on $V_{\varepsilon}$ concentrate near points of the $x_{n}$ axis, as $\varepsilon \rightarrow 0$, while a function $u_{\varepsilon}$ which minimizes $f_{\varepsilon}$ on $M_{\varepsilon}$ concentrates near a sphere of codimension 2; the arguments used in this section for the proof of Theorem 2.1 show that $F_{\varepsilon}\left(u_{\varepsilon}\right)$ behaves as $\varepsilon^{2}$ when $\varepsilon \rightarrow 0$; on the contrary, $\min _{V_{\varepsilon}} F_{\varepsilon}$ behaves as $\varepsilon^{n}$, as one can verify in the following way. Let $\bar{x}=\left(0, \ldots, 0, \bar{x}_{n}\right)$ be a point of the $x_{n}$-axis belonging to $\Omega$; choose a function $\bar{u} \in C_{0}^{\infty}(B(0,1))$ radially symmetric with respect to the origin, $\bar{u}^{-} \equiv 0, \bar{u}^{+} \not \equiv 0$, and, for all $\varepsilon>0$, consider the function $\bar{u}_{\varepsilon}$ defined by $\bar{u}_{\varepsilon}(x)=\bar{u}\left(\frac{x-\bar{x}}{\varepsilon}\right)(\bar{u}$ is extended by the value zero outside $B(0,1))$; for $\varepsilon>0$ small enough, $\bar{u}_{\varepsilon} \in H_{S}(\Omega)$ and there exists a unique $\bar{t}_{\varepsilon}>0$ such that $\bar{t}_{\varepsilon} \bar{u}_{\varepsilon} \in V_{\varepsilon}$; then, a direct computation shows that $\lim _{\varepsilon \rightarrow 0} \varepsilon^{-n} F_{\varepsilon}\left(\bar{t}_{\varepsilon} \bar{u}_{\varepsilon}\right)<+\infty$.

If $\Omega$ meets the $x_{n}$-axis and $p=\frac{2 n}{n-2}$, then the infimum $\inf _{V_{\varepsilon}} F_{\varepsilon}$ is not achieved for any $\varepsilon>0$. In fact, if $\bar{x}$ is a point of the $x_{n}$-axis belonging to $\Omega$, we can consider the functions $\tilde{u}_{\delta} \in H_{S}(\Omega)$ defined by

$$
\tilde{u}_{\delta}(x)=\zeta(x)\left[\delta^{2}+|x-\bar{x}|^{2}\right]^{(2-n) / 2} \quad \forall x \in \Omega,
$$

where $\zeta \in C_{0}^{\infty}(\Omega) \cap H_{S}(\Omega)$ is a cut-off function, $0 \leqslant \zeta \leqslant 1, \zeta \equiv 1$ in a neighbourhood of $\bar{x}$. Notice that, for all $\delta>0$ and $\varepsilon>0$, there exists $\tilde{t}_{\delta, \varepsilon}>0$ such that $\tilde{t}_{\delta, \varepsilon} \tilde{u}_{\delta} \in V_{\varepsilon}$. Since the functions $\left[\delta^{2}+|x-\bar{x}|^{2}\right]^{(2-n) / 2}$ are minimizing functions for the best Sobolev constant (see [27]), letting $\delta \rightarrow 0$ one can see that $\inf _{V_{\varepsilon}} F_{\varepsilon}$ is related to the best Sobolev constant and, arguing for example as in [4,5], one can prove that $\inf _{V_{\varepsilon}} F_{\varepsilon}$ cannot be achieved for any $\varepsilon>0$ because of the nonexistence result of Pohožaev (see [22]). However, even if the infimum is not achieved, for $p=\frac{2 n}{n-2}$, under suitable assumptions on $\Omega$, one can find critical points for $F_{\varepsilon}$ constrained on $V_{\varepsilon}$ as local minimum points arguing as in [18]. On the contrary, when $p>\frac{2 n}{n-2}$ and $\Omega$ meets the $x_{n}$-axis, $F_{\varepsilon}$ constrained on $V_{\varepsilon}$ does not admit neither minimum, nor local minimum points (this difficulty has been first pointed out in [21]). In fact, $\inf _{V_{\varepsilon}} F_{\varepsilon}=0$, as one can verify in the following way: consider, as above, the functions $\bar{u}_{\delta}(x)=\bar{u}\left(\frac{x-\bar{x}}{\delta}\right)$, which belong to $H_{S}(\Omega)$ for $\delta>0$ small enough; then, a direct computation shows that, for all $\varepsilon>0$, there exists a unique $\bar{t}_{\delta, \varepsilon}>0$ such that $\bar{t}_{\delta, \varepsilon} \bar{u}_{\delta} \in V_{\varepsilon}$ and (since $p>\frac{2 n}{n-2}$ ) $\lim _{\delta \rightarrow 0} F_{\varepsilon}\left(\bar{t}_{\delta, \varepsilon} \bar{u}_{\delta}\right)=0$.

In order to prove that $F_{\varepsilon}$ constrained on $V_{\varepsilon}$ does not have any local minimum point, it suffices to prove that for all $u \in V_{\varepsilon}$ there exists a sequence $\left(u_{i}\right)_{i}$ in $V_{\varepsilon}$, which converges to $u$ in $H_{0}^{1,2}(\Omega)$ and in $L^{p}(\Omega)$, such that $F_{\varepsilon}\left(u_{i}\right)<F_{\varepsilon}(u)$ for all $i \in \mathbb{N}$.

In order to find such a sequence, let us consider the functions

$$
\bar{u}_{i, \delta}=\left(1-\frac{1}{i}\right)^{1 / p} u+\left(\frac{1}{i}\right)^{p} \frac{\|u\|_{L^{p}(\Omega)}}{\left\|\bar{u}_{\delta}\right\|_{L^{p}(\Omega)}} \bar{u}_{\delta}
$$

and $u_{i, \delta}=\bar{t}_{\varepsilon, i, \delta} \bar{u}_{i, \delta}$, where $\bar{u}_{\delta}$ is defined as above and $\bar{t}_{\varepsilon, i, \delta}$ is the unique positive number such that $\bar{t}_{\varepsilon, i, \delta} \bar{u}_{i, \delta} \in V_{\varepsilon}$. One can verify that

$$
\lim _{\delta \rightarrow 0} \int_{\Omega} \bar{u}_{i, \delta}^{p} \mathrm{~d} x=\int_{\Omega} u^{p} \mathrm{~d} x \quad \forall i \in \mathbb{N}
$$


and, since $p>\frac{2 n}{n-2}$,

$$
\begin{aligned}
\lim _{\delta \rightarrow 0} \int_{\Omega}\left(\varepsilon^{2}\left|D \bar{u}_{i, \delta}\right|^{2}+\bar{u}_{i, \delta}^{2}\right) \mathrm{d} x & =\left(1-\frac{1}{i}\right)^{2 / p} \int_{\Omega}\left(\varepsilon^{2}|D u|^{2}+u^{2}\right) \mathrm{d} x \\
& <\int_{\Omega}\left(\varepsilon^{2}|D u|^{2}+u^{2}\right) \mathrm{d} x \quad \forall i \in \mathbb{N},
\end{aligned}
$$

where the strict inequality holds because $u \neq \equiv 0$ in $\Omega$ (as $u \in V_{\varepsilon}$ ). It follows that there exists a sequence of positive numbers $\delta_{i} \rightarrow 0$ such that, if we set $u_{i}=\bar{t}_{\varepsilon, i, \delta_{i}} \bar{u}_{i, \delta_{i}}$, then the sequence $\left(u_{i}\right)_{i}$ satisfies the desired properties.

\section{References}

[1] A. Bahri, J.M. Coron, On a nonlinear elliptic equation involving the critical Sobolev exponent: the effect of the topology of the domain, Comm. Pure Appl. Math. 41 (1988) 253-294.

[2] A. Bahri, Y.Y. Li, O. Rey, On a variational problem with lack of compactness: the topological effect of the critical points at infinity, Calc. Var. 3 (1) (1995) 67-93.

[3] H. Berestycki, P.L. Lions, Nonlinear scalar fields equations - I. Existence of a ground-state, Arch. Rational Mech. Anal. 82 (1983) $313-$ 346.

[4] H. Brézis, Elliptic equations with limiting Sobolev exponents - the impact of topology, Comm. Pure Appl. Math. 39 (suppl.) (1986) S17-S39.

[5] H. Brézis, L. Nirenberg, Positive solutions of nonlinear elliptic equations involving critical Sobolev exponents, Comm. Pure Appl. Math. 36 (4) (1983) 437-477.

[6] J.M. Coron, Topologie et cas limite des injections de Sobolev, C. R. Acad. Sci. Paris Sér. I Math. 299 (7) (1984) $209-212$.

[7] E.N. Dancer, K. Zhang, Uniqueness of solutions for some elliptic equations and systems in nearly star-shaped domains, Nonlinear Anal. Ser. A $41(5 / 6)(2000) 745-761$.

[8] B. Gidas, W.M. Ni, L. Nirenberg, Symmetry of positive solutions of nonlinear elliptic equations in $\mathbb{R}^{N}$, in: Mathematical Analysis and Applications - Part A, Advances in Mathematics Supplementary Studies, vol. 7-A, Academic Press, 1981, pp. 369-402.

[9] M.K. Kwong, Uniqueness of positive solutions of $\Delta u-u+u^{p}=0$, Arch. Rational Mech. Anal. 105 (1989) 243-266.

[10] P.L. Lions, The concentration-compactness principle in the calculus of variations. The locally compact case. I, Ann. Inst. H. Poincaré Anal. Non Linéaire 1 (2) (1984) 109-145.

[11] W. Littman, G. Stampacchia, H.F. Weinberger, Regular points for elliptic equations with discontinuous coefficients, Ann. Scuola Norm. Sup. Pisa 17 (3) (1963) 43-77.

[12] R. Molle, D. Passaseo, Positive solutions for slightly super-critical elliptic equations in contractible domains, Preprint Dip. Matem. Univ. Lecce, n. 6, 2001;

R. Molle, D. Passaseo, C. R. Acad. Sci. Paris, Sér. I Math. 335 (5) (2002) 459-462.

[13] R. Molle, D. Passaseo, Nonlinear elliptic equations with critical Sobolev exponent in nearly starshaped domains, C. R. Acad. Sci. Paris, Sér. I Math. 335 (12) (2002) 1029-1032.

[14] R. Molle, D. Passaseo, Positive solutions of slightly supercritical elliptic equations in symmetric domains, Ann. Inst. H. Poincaré Anal. Non Linéaire 21 (5) (2004) 639-656.

[15] R. Molle, D. Passaseo, Multispike solutions of nonlinear elliptic equations with critical Sobolev exponent, Preprint del Dipartimento di Matematica dell'Università di Roma "Tor Vergata”, 2003.

[16] R. Molle, D. Passaseo, On the existence of positive solutions of slightly supercritical elliptic equations, Adv. Nonlinear Stud. 3 (3) (2003) 301-326.

[17] R. Molle, D. Passaseo, Nonlinear elliptic equations with large supercritical exponents, Preprint del Dipartimento di Matematica dell'Università di Roma "Tor Vergata", 2003.

[18] D. Passaseo, Multiplicity of positive solutions of nonlinear elliptic equations with critical Sobolev exponent in some contractible domains, Manuscripta Math. 65 (2) (1989) 147-165.

[19] D. Passaseo, Nonexistence results for elliptic problems with supercritical nonlinearity in nontrivial domains, J. Funct. Anal. 114 (1) (1993) 97-105.

[20] D. Passaseo, New nonexistence results for elliptic equations with supercritical nonlinearity, Differential Integral Equations 8 (3) (1995) 577-586.

[21] D. Passaseo, Nontrivial solutions of elliptic equations with supercritical exponent in contractible domains, Duke Math. J. 92 (2) (1998) 429-457. 
[22] S.I. Pohožaev, On the eigenfunctions of the equation $\Delta u+\lambda f(u)=0$, Soviet Math. Dokl. 6 (1965) 1408-1411.

[23] O. Rey, The role of the Green's function in a nonlinear elliptic equation involving the critical Sobolev exponent, J. Funct. Anal. 89 (1) (1990) 1-52.

[24] F. Riesz, B.Sz. Nagy, Functional Analysis, Dover, New York, 1990.

[25] R. Schaaf, Uniqueness for semilinear elliptic problems: supercritical growth and domain geometry, Adv. Differential Equations 5 (10-12) (2000) 1201-1220.

[26] W.A. Strauss, Existence of solitary waves in higher dimensions, Comm. Math. Phys. 55 (1977) 149-162.

[27] G. Talenti, Best constants in Sobolev inequality, Ann. Mat. Pura Appl. 110 (1976) 353-372. 